\title{
Activation of Focal Adhesion Kinase and Src Mediates Acquired Sorafenib Resistance in A549 Human Lung Adenocarcinoma Xenografts $[$ [
}

\author{
Qingyu Zhou, ${ }^{1}$ Xiaofang Guo, and Riya Choksi
}

Department of Pharmaceutical Sciences, College of Pharmacy, University of South Florida, Tampa, Florida

Received January 31, 2017; accepted October 2, 2017

\begin{abstract}
Despite encouraging clinical results with sorafenib monotherapy in patients with KRAS-mutant non-small-cell lung cancer (NSCLC), the overall survival benefit of this drug is limited by the inevitable development of acquired resistance. The exact mechanism underlying acquired sorafenib resistance in KRAS-mutant NSCLC is unclear. In this study, the mechanism of acquired sorafenib resistance was explored using a biologically relevant xenograft model, which was established by using the A549 human lung adenocarcinoma cell line and an in vivo-derived, sorafenib-resistant A549 subline (A549/SRFres). Results from the initial study demonstrated that sorafenib treatment significantly decreased E-cadherin $(P<0.05)$ levels but significantly increased matrix metallopeptidase 9 (MMP9) levels $(P<0.01)$ in A549/SRFres tumors, whereas expression levels of phosphoprotein kinase $B(A K T)$, phospho-focal adhesion kinase (FAK), and phospho-Src were elevated in sorafenib-treated A549 and
\end{abstract}

A549/SRFres tumors. We next examined whether concomitant dasatinib treatment could overcome acquired sorafenib resistance by blocking the FAK/Src escape route that mediates resistance. Despite the observed in vitro synergy between sorafenib and dasatinib, the in vivo antitumor effect of half-dose sorafenib-dasatinib combination therapy was inferior to that of the full-dose sorafenib treatment. Although the sorafenib-dasatinib combination effectively inhibited Src and AKT phosphorylation, it did not block the Y576/577-FAK phosphorylation, nor did it decrease vimentin protein expression; unexpectedly, it increased Y397-FAK phosphorylation and MMP9 protein expression in tumors. These results suggest that acquired sorafenib resistance in KRAS-mutant A549 xenografts involves the compensatory activation of FAK and Src, and Src inhibition alone is insufficient to diminish sorafenib-promoted epithelial-mesenchymal transition process and invasive potentials in tumors.

\section{Introduction}

Over the past two decades, the therapeutic landscape of advanced cancer has been revolutionized by the rapid development of molecularly targeted therapy. With the increasing use of targeted therapeutic agents came the challenge of addressing the inevitable development of acquired drug resistance. In the field of targeted therapy in non-small-cell lung cancer (NSCLC), much effort has focused on developing inhibitors of epidermal growth factor receptor (EGFR) as the first-line treatment of patients with activating EGFR mutations (Pao and Chmielecki, 2010) and identifying the mechanisms of acquired resistance to the EGFR inhibitors so that therapeutic approaches can be

This work was supported by the startup fund and internal seed grant provided to Q.Z. by the University of South Florida College of Pharmacy.

${ }^{1}$ Current affiliation: College of Pharmacy, University of South Florida, Tampa, Florida.

doi.org/10.1124/jpet.117.240507.

S This article has supplemental material available at jpet.aspetjournals.org. developed to overcome the resistance (Wheeler et al., 2010). Besides EGFR mutations, the aberrant activation of the RAS/RAF/MEK/ extracellular signal-regulated protein kinases (ERK) pathway via activating mutations in KRAS also occurs frequently in NSCLC, mainly adenocarcinomas (Götz, 2008). It has been reported that the prevalence of patients with KRAS-mutant NSCLC is approximately $15 \%$ (Chan and Hughes, 2015). Since direct inhibition of KRAS remains therapeutically challenging, an alternative approach for treating KRAS-mutant NSCLC has been pursued that targets signaling pathways downstream of RAS. In this regard, several recent clinical trials have been conducted to examine the efficacy of sorafenib, a multikinase inhibitor targeting the RAF/MEK/ERK pathway and the vascular endothelial growth factor receptors (VEGFRs) and plateletderived growth factor receptors (PDGFRs) (Wilhelm et al., 2006), in the treatment of NSCLC.

In the BATTLE (biomarker-integrated approaches of targeted therapy for lung cancer elimination) trial, which

ABBREVIATIONS: AKT, protein kinase B; Cl, combination index; DCR, disease control rate; DMSO, dimethylsulfoxide; EGFR, epidermal growth factor receptor; EMT, epithelial-mesenchymal transition; ERK1/2, extracellular signal-regulated protein kinases 1 and 2; FA, fraction affected; FAK, focal adhesion kinase; FGF, fibroblast growth factor; GSK $3 \alpha / \beta$, glycogen synthase kinases $3 \alpha$ and $3 \beta$; HCC, hepatocellular carcinoma; HPLC, highperformance liquid chromatography; IS, internal standard; MAPK, mitogen-activated protein kinase; MCL-1, myeloid cell leukemia sequence 1 protein; MMP9, matrix metallopeptidase 9; mTOR, mechanistic target of rapamycin; MTT, 3-[4,5-dimethylthiazole-2-yl]-2,5-diphenyl-tetrazolium bromide; MVD, microvessel density; NSCLC, non-small-cell lung cancer; PDGFR, platelet-derived growth factor receptor; RSK, 90-kDa ribosomal S6 kinase; $\alpha$-SMA, $\alpha$-smooth muscle actin; VEGFR, vascular endothelial growth factor receptor. 
was designed to evaluate the effectiveness of small-molecule kinase inhibitors in patients with chemorefractory NSCLC based on relevant molecular biomarkers (Kim et al., 2011), sorafenib treatment resulted in a better-than-average overall 8-week disease control rate (DCR) (58\% vs. $46 \%)$ in the KRAS/BRAF, VEGF/VEGFR-2, and no-marker groups. More strikingly, sorafenib had a $79 \%$ DCR compared with a $14 \%$ DCR with erlotinib in the KRAS / BRAF marker group (Kim et al., 2011); however, results from the phase 3 MISSION (Monotherapy admInistration of Sorafenib in patientS wIth nOn-small cell luNg cancer) trial, in which sorafenib was used as a single agent in patients with advanced relapsed or refractory NSCLC, indicated that sorafenib monotherapy did not improve the overall survival in spite of a statistically significant improvement of several secondary endpoints, including progression-free survival and time to disease progression (Paz-Ares et al., 2015). Collectively, these clinical data suggest that the initial treatment with sorafenib is efficacious, but resistance to the drug is eventually developed. Given the acquired resistance being a major factor that limits the clinical success of sorafenib therapy in patients with KRAS-mutant NSCLC, understanding the molecular mechanisms underlying the resistance is crucial for maximizing the therapeutic outcome.

Acquired sorafenib resistance has been associated with the activation of bypass signaling driven by alternative receptor tyrosine kinases. A diversity of adaptive molecular events underpinning the resistance have been reported under different experimental conditions, including the induction of metallothionein-1G (Sun et al., 2016) and the activation of parallel pathways that promote the malignant properties of the tumor, such as the protein kinase B (AKT) pathway (Chen et al., 2011; Morgillo et al., 2011; Lindblad et al., 2016), the mitogen-activated protein kinase (MAPK) pathway (Morgillo et al., 2011; Harada et al., 2014), the signal transducer and activator of transcription 3 pathway (Tai et al., 2012), the hepatocyte growth factor/c-Met pathway (Firtina Karagonlar et al., 2016), and the insulin-like growth factor/fibroblast growth factor (FGF) pathway (Tovar et al., 2017).

In the present study, we sought to examine the molecular mechanisms by which NSCLC adapts to evade sorafenib therapy using a biologically relevant xenograft model of acquired sorafenib resistance and to translate the knowledge into a combinatorial therapy option comprising sorafenib and dasatinib with the goal of increasing the antitumor efficacy of sorafenib against tumors with acquired resistance to sorafenib and elucidate underlying mechanisms of interaction between sorafenib and dasatinib. Dasatinib is a potent kinase inhibitor targeting multiple protein kinases, including, Bcr-Abl, Src, stem cell factor receptor, FGF receptor 1 , and PDGFR $\beta$ (Kantarjian et al., 2006). Combination therapy with sorafenib and dasatinib for the treatment of NSCLC has not been described so far. By using an A549 human lung adenocarcinoma xenograft model, we were able to explore the mechanisms of acquired sorafenib resistance in the context of tumor microenvironment, which is known to contribute substantially to the acquisition of tumor resistance to targeted therapies (McMillin et al., 2013). The goal of this investigation is not only to gain a broader understanding of acquired sorafenib resistance in NSCLC but also to provide a framework for the rational design of targeted combinatorial therapy that can effectively manage such resistance.

\section{Materials and Methods}

\section{Reagents}

Sorafenib base, sorafenib tosylate, and dasatinib base were purchased from LC Laboratories (Woburn, MA). For the in vitro study, sorafenib base and dasatinib base were dissolved in dimethylsulfoxide (DMSO). To prepare the stock solutions for the in vivo study, sorafenib tosylate was dissolved in Cremophor EL/ethanol (50:50, v/v) at $4 \times$ concentration, and dasatinib base was dissolved in propylene glycol at $2 \times$ concentration. The stock solutions were prepared fresh every 3 days. The final dosing solution was prepared by diluting the stock solution to $1 \times$ concentration with sterile water. All other chemicals, solvents, and reagents were obtained from commercial sources.

\section{Animals}

Male athymic nude mice (Hsd: Athymic Nude-Foxn $1^{n u} ; 6-8$ weeks old) were purchased from Envigo (Indianapolis, IN). All animal experiments were approved by the Institutional Animal Care and Use Committee and performed according to the National Institutes of Health guidelines.

\section{Cell Line, Sorafenib-Resistant Subclone, and Culture Condition}

The A549 human lung adenocarcinoma cell line (ATCC CCL-185) was purchased from the American Type Culture Collection (Manassas, VA). The sorafenib-resistant A549 subclone (A549/SRFres) was derived from a sorafenib-treated animal with the fastest growing A549 xenograft. Both parental A549 and A549/SRFres cells were cultured in a mixture of Dulbecco's modified Eagle's media/Ham's F12 at a ratio of 1:1 (Mediatech Inc., Herndon, VA) supplemented with $10 \%$ fetal bovine serum (Sigma-Aldrich, St. Louis, MO), $100 \mathrm{U} / \mathrm{ml}$ penicillin, and $100 \mu \mathrm{g} / \mathrm{ml}$ streptomycin, maintained in a humidified atmosphere of $5 \% \mathrm{CO}_{2}$ in air at $37^{\circ} \mathrm{C}$. All experiments were conducted using A549 and A549/SRFres cells with passage numbers less than 20 and 10, respectively.

\section{In Vivo Sorafenib-Resistant A549 Xenograft Model}

Initial Model Development Protocol. Parental A549 tumor cells $\left(5 \times 10^{6}\right)$ suspended in $0.2 \mathrm{ml}$ of Matrigel (BD Biosciences, San Jose, CA) were injected subcutaneously into the left flank of the athymic nude mice. Tumor-bearing mice were randomly divided into control $(n=9)$ and sorafenib $(n=10)$ groups. Seven days after tumor inoculation, each animal was given once-daily oral administration of either vehicle or sorafenib on a 6-days-on/1-day-off schedule for 15 consecutive weeks. Sorafenib was given at a dose of $10 \mathrm{mg} / \mathrm{kg}$ per day for the first week, $20 \mathrm{mg} / \mathrm{kg}$ per day for the second week, and $40 \mathrm{mg} / \mathrm{kg}$ per day from the third week onward. Tumor growth was monitored once a week using a digital caliper (Fisher Scientific, Hampton, NH) with the volume calculated as $0.5 \times$ length $\times$ width $^{2}$. Phenotypic sensitivity of individual mice to sorafenib treatment was defined based on the degree of suppression of tumor growth, which was expressed as the tumor growth index (i.e., the ratio of tumor volume on the last day to that on the first day of the treatment).

In Vivo Study Protocol. Each animal was inoculated subcutaneously with A549 and A549/SRFres cells $\left(5 \times 10^{6}\right.$ cells for each xenograft) on the left and right flanks, respectively (Fig. 1C). Seven days after tumor inoculation, tumor-bearing animals were randomly divided into control $(n=6)$ and sorafenib $(n=7)$ groups. Each animal received once-daily oral administration of vehicle control or sorafenib on a 6-days-on/1-day-off schedule for 8-12 consecutive weeks. Sorafenib dose-escalation scheme was the same as that described already. Tumor growth was determined as described. All animals were euthanized with $\mathrm{CO}_{2}$ gas at the end of the treatment period. The tumor mass was excised, snap-frozen on dry ice, and stored at $-80^{\circ} \mathrm{C}$ before subjected to Western blot analysis. 


\section{In Vitro Comparison of Doubling Time between A549 and A549/SRFres Cells}

See the Supplemental Methods for a detailed description of the experiment.

\section{Wound-Healing Assay}

See the Supplemental Methods for a detailed description of the assay.

\section{Cell Invasion Assay}

See the Supplemental Methods for a detailed description of the assay.

\section{In Vitro Cytotoxicity Assay}

Cytotoxicity was analyzed using the MTT (3-[4,5-dimethylthiazole2-yl]-2,5-diphenyl-tetrazolium bromide) assay. Briefly, A549 and A549/SRFres cells were seeded in 96-well plates at a density of $3 \times$ $10^{3}$ cells/well and allowed to attach overnight. On the next day, culture media containing vehicle control (0.5\% DMSO), sorafenib (13 nM-100 $\mu \mathrm{M})$, dasatinib (13 $\mathrm{nM}-100 \mu \mathrm{M})$, or the combination of sorafenib and dasatinib ( $6 \mathrm{nM}-50 \mu \mathrm{M}$ for each drug) were added to the appropriate wells. The molar ratios of sorafenib to dasatinib were fixed at 1:1, 3:1, and 1:3. After the cells were treated for 72 hours, $5 \mu \mathrm{l}$ of $5 \mathrm{mg} / \mathrm{ml}$ of MTT in phosphate-buffered saline was added to each well, and individual plates were incubated for 2 hours at $37^{\circ} \mathrm{C}$, followed by the addition of $100 \mu \mathrm{l}$ of DMSO to each well and incubation at room temperature in the dark for 2 more hours. Optical densities were measured at $570 \mathrm{~nm}$ with a SpectraMax 190 microplate reader equipped with SoftMax Pro software (Molecular Devices, Sunnyvale, CA). The growth of treated cells was expressed as a percentage of vehicle control cultures. Concentrations of individual drugs required for $50 \%$ inhibition of cell growth (i.e., $\mathrm{IC}_{50}$ ) compared with control cells were calculated by nonlinear fitting of the experimental data obtained from multiple independent experiments performed in duplicate or triplicate using the GraphPad Prism 5.0 program (GraphPad Software, Inc. La Jolla, CA). The combination effects of sorafenib and dasatinib was further evaluated based on the combination index (CI) values (Chou and Talalay, 1981).

\section{In Vivo Evaluation of Combination Therapy with Sorafenib and Dasatinib}

Three weeks after individual athymic nude mice were injected subcutaneously with A549 and A549/SRFres cells $\left(5 \times 10^{6}\right)$ on the left and right franks, respectively, those tumor-bearing animals were randomly divided into four groups: 1$)$ vehicle control $(n=7), 2)$ fulldose sorafenib (40 mg/kg per day, $n=7)$, 3) full-dose dasatinib ( $40 \mathrm{mg} / \mathrm{kg}$ per day, $n=8)$, and 4 ) half-dose combination $(20 \mathrm{mg} / \mathrm{kg}$ per day of sorafenib and $20 \mathrm{mg} / \mathrm{kg}$ of dasatinib, $n=8$ ) groups. Individual animals were given once-daily oral administration of either vehicle or therapeutic agents for 28 consecutive days. Body weight and tumor volume were measured twice a week throughout the treatment period. On the last day of the treatment period, 4 hours after the last dose, individual animals were sedated with isoflurane and euthanized with terminal bleeding from the vena cava. Plasma was separated by centrifugation and then stored at $-80^{\circ} \mathrm{C}$ before being subjected to drug analysis using the high-performance liquid chromatography (HPLC). The tumor mass was immediately excised, snap-frozen on dry ice, and stored at $-80^{\circ} \mathrm{C}$ before being subjected to drug analysis, Western blot analysis, and immunofluorescence double staining.

\section{Immunofluorescence Double Staining}

Frozen A549 and A549/SRFres tumor samples were cryosectioned at a thickness of $10 \mu \mathrm{m}$ and then subjected to immunofluorescence double staining as described previously (Zhou et al., 2012). The primary antibodies used included rabbit anti-mouse collagen type IV antibody (1:100; EMD Millipore, Billerica, MA ), rabbit monoclonal antibody anti-E-cadherin (1:100; Cell Signaling Technologies, Danvers, MA), rabbit monoclonal anti-Ki-67 (1:100; Abcam, Cambridge, MA), rabbit polyclonal anti- $\alpha$-smooth muscle actin ( $\alpha$-SMA) (1:100; Abcam), and rat monoclonal anti-CD31 (1:200; BD Pharmingen, San Jose, CA). Secondary antibodies were Alexa Fluor 594 conjugated goat anti-rabbit IgG and Alexa Fluor 488 conjugated goat anti-rat IgG (1: 200 for each; Thermo Fisher Scientific, Waltham, MA), which generate red and green fluorescence, respectively. Sections were mounted with ProLong Gold Antifade Reagent with DAPI (Life Technologies, Carlsbad, CA) and analyzed under an Olympus BX53 digital fluorescence microscope. Images were processed using the ImageJ 1.47 software (from the National Institutes of Health and available at http://rsb.info.nih.gov/ij/).

\section{Western Blot Analysis}

In vitro cultured parental A549 and A549/SRFres cells treated with vehicle $(0.5 \%$ DMSO), sorafenib $(10 \mu \mathrm{M})$, and dasatinib $(0.2$ or 0.02 $\mu \mathrm{M})$ alone or in combination for 4 hours and tumor tissue samples collected from the in vivo studies were subjected to Western blot analysis. Cell lysate samples and tumor tissue lysate samples were prepared as previously described (Zhou et al., 2008). Immunoblotting was carried out with the following primary antibodies: rabbit polyclonal antibodies from Abcam: ATP-binding cassette subfamily G member 2 (1:1000), $\alpha$-SMA (1:1000), matrix metallopeptidase 9 (MMP9; 1:5000), rabbit polyclonal or monoclonal antibodies from Cell Signaling Technologies (1:1000 dilution for all): AKT (pan), phosphor-AKT (S473), extracellular signal-regulated protein kinases 1 and 2 (ERK1/2), phospho-ERK1/2 (T202/Y204), focal adhesion kinase (FAK), phospho-FAK (Y397), phospho-FAK(Y576/577), glycogen synthase kinases $3 \alpha$ and $3 \beta($ GSK $3 \alpha / \beta)$, phospho-GSK $3 \alpha / \beta$ (S21/9), MEK1/2, phospho-MEK1/2 (S217/221), mechanistic target of rapamycin (mTOR), phospho-mTOR (S2448), 90-kDa ribosomal S6 kinase 1/2/3 (RSK1/2/3), phospho-p90RSK (S380), Src, phospho-Src (Y416), myeloid cell leukemia sequence 1 protein (MCL-1), $\beta$-catenin, E-cadherin, and vimentin. Blots were incubated with horseradish peroxidase-conjugated secondary antibodies (1:15,000; Santa Cruz) and immunoreactive protein bands were visualized by the enhanced chemiluminescence system (PerkinElmer, Waltham, MA). The membrane was then stripped and reprobed with $\beta$-actin (1:4000; SigmaAldrich) as a loading control. Band areas of individual proteins were quantified using the ImageJ software (https://imagej.nih.gov/ij/). Normalization for loading differences was achieved by dividing the densitometry values for individual proteins by the densitometry values for $\beta$-actin in the same lane. Protein expression levels in the drug-treated tumors were expressed as relative to those in the control tumors. For phosphorylated proteins, the signal values were expressed as the ratio of phosphorylated to total species relative to that of the control tumors.

\section{Quantitative Determination of Dasatinib and Sorafenib Concentrations in Plasma and Tumors}

We developed and validated an HPLC method for simultaneous determination of dasatinib and sorafenib in mouse plasma and xenograft tumor tissues. Plasma and tumor tissue homogenate (tissue:MilliQ water $=1: 9, \mathrm{w} / \mathrm{v}$ ) samples were deproteinated by adding three volumes of methanol containing $5 \mu \mathrm{g} / \mathrm{ml}$ of 7-hydroxywarfarin (the internal standard, IS), followed by centrifugation at 13,000 rpm for 10 minutes. Ten-microliter aliquots of the supernatants were injected onto the reversed-phase HPLC system with a diode array detector. The chromatographic separation was achieved on an octadecylsilane bonded silica column (Luna $3 \mu \mathrm{m} \mathrm{C18,}$ $50 \times 4.6 \mathrm{~mm}$; Phenomenex) at room temperature. Gradient elution comprised $20 \%$ acetonitrile containing $10 \mathrm{mM}$ ammonium acetate and $0.1 \%$ formic acid as solvent $\mathrm{A}$ and $60 \%$ acetonitrile containing $10 \mathrm{mM}$ 
A

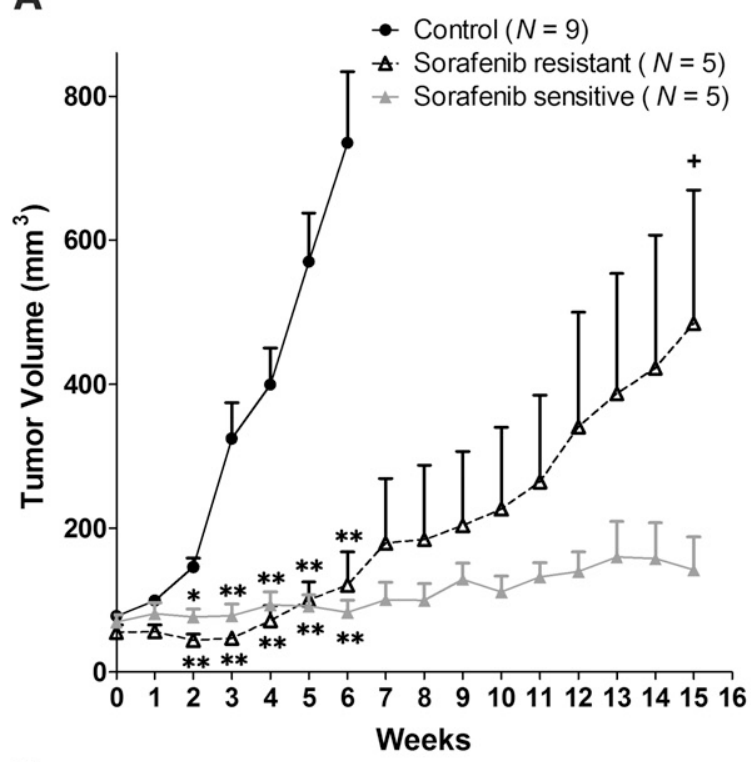

B

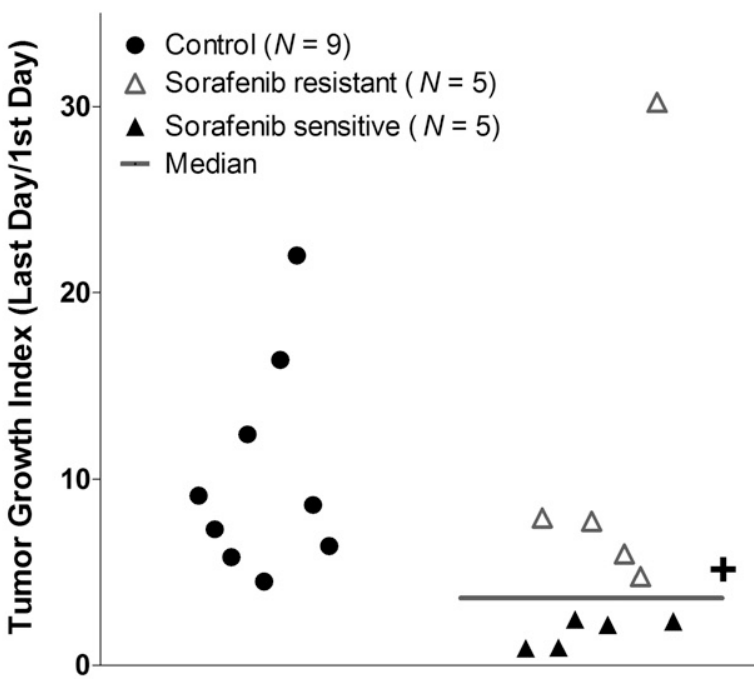

C

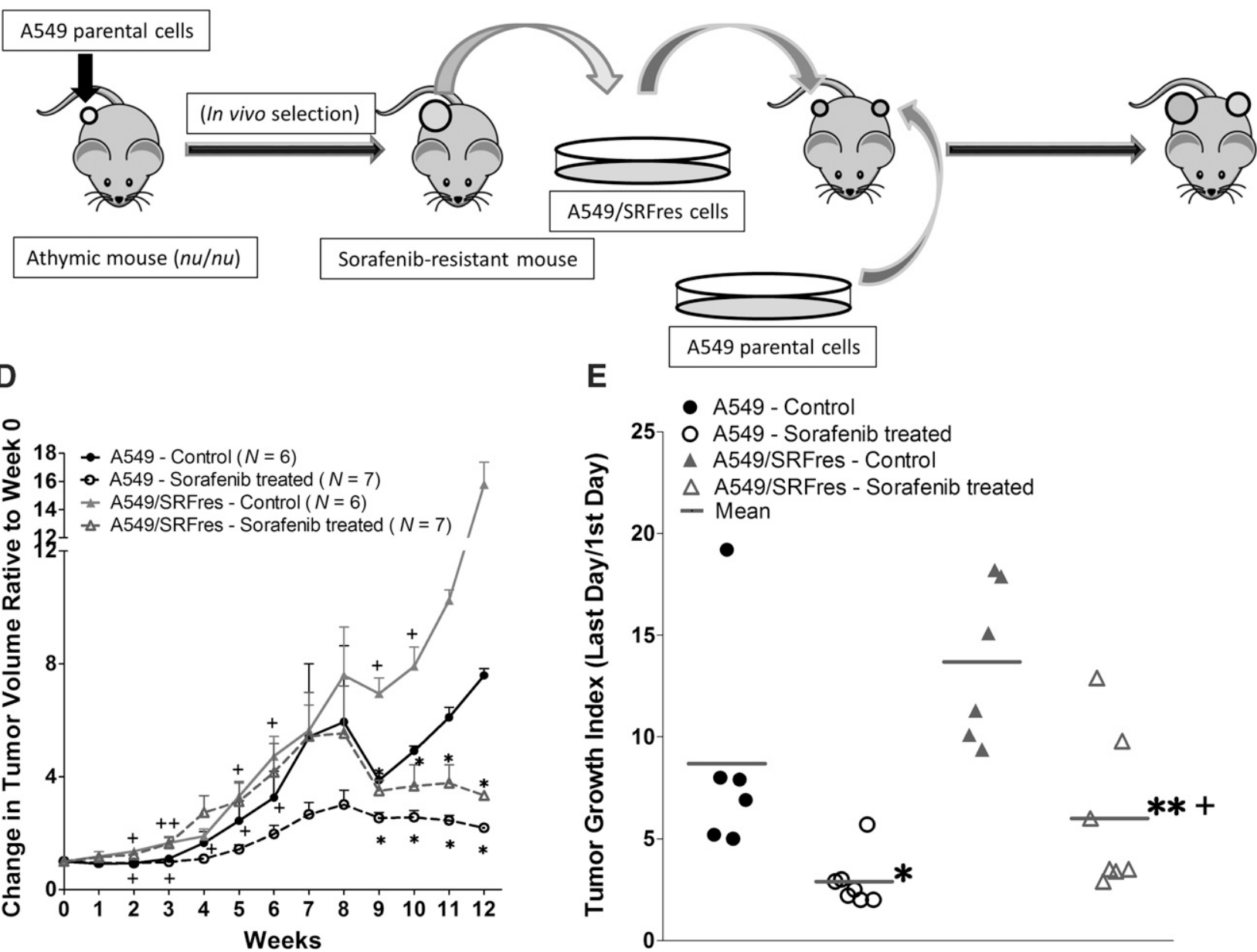

Fig 1 In vivo selection of A549 human lung adenocarcinoma cells resistant to sorafenib treatment and evaluation of the sorafenib-resistant A549 xenograft model. (A) Differential tumor growth rates are observed in A549 tumor-bearing mice treated with vehicle control for 6 weeks ( $\mathrm{n}=9$ ) or $40 \mathrm{mg} / \mathrm{kg}$ of sorafenib once daily for 15 weeks $(n=10)$. Sorafenib-sensitive and -resistant groups are classified based on the tumor growth index value, calculated as the ratio of tumor volume at the first day of treatment to that at the last day of treatment in those with a tumor growth index value greater than the median value of 3.6, are classified as sorafenib-resistant animals $(n=5)$; the remainder are defined as sorafenib-sensitive animals $(n=5)$. $(B)$ Tumor volumes of individual study groups. (C) Experimental design for the in vivo study that compared the antitumor activities of sorafenib in A549 and sorafenib-resistant A549 xenografts. The sorafenib-resistant A549 subclone (A549/SRFres) was derived from a sorafenib-treated animal with the fastest growing A549 xenograft. (D) Sorafenib treatment inhibits the tumor growth in both A549 and A549/SRFres xenografts. Individual animals bearing both 
ammonium acetate and $0.1 \%$ formic acid as solvent $\mathrm{B}$, with a linear gradient increasing from $0 \%$ to $100 \%$ B from 1 to 3 minutes, maintaining $100 \%$ B from 3 to 9 minutes, decreasing from $100 \%$ to $0 \% \mathrm{~B}$ from 9 to 11 minutes. The total run time was 14 minutes. Dasatinib and IS were best detected at $325 \mathrm{~nm}$, and sorafenib was best detected at $255 \mathrm{~nm}$. Retention time was about 5.8, 6.7, and 8.4 minutes for dasatinib, IS, and sorafenib, respectively. Standard curves of dasatinib and sorafenib were linear within the ranges of $62-5000 \mathrm{ng} / \mathrm{ml}\left(r^{2}>\right.$ 0.99) in plasma and tumor homogenates, respectively. The lower limits of quantitation in plasma and tumor tissue homogenates were $62 \mathrm{ng} / \mathrm{ml}$ for both dasatinib and sorafenib.

\section{Statistical Analyses}

Statistical analyses were performed using Number Cruncher Statistical Systems 2007 (Keysville, UT). Data are presented as the mean \pm S.D. unless otherwise indicated. Comparison of means from two matched groups was made using the paired-sample $t$ test. Comparison of means between two independent groups was made using the independent sample $t$ test. In case of multiple comparisons, Kruskal-Wallis one-way analysis of variance on ranks, followed by the post hoc Kruskal-Wallis multiple comparison $z$-value test, was used. Pearson correlations were used to describe relations between two variables. A two-sided $P$ value less than 0.05 was considered statistically significant.

\section{Results}

In Vivo Selection of Sorafenib-Resistant A549 Xenograft Line. Clinical trials have shown that patients with EGFR wild-type and KRAS-mutant NSCLC benefit from sorafenib treatment (Blumenschein et al., 2013; Dingemans et al., 2013); therefore, the A549 human adenocarcinoma cell line expressing wild-type EGFR and harboring a G12S KRAS mutation (Mahoney et al., 2009) was used to establish the in vivo sorafenib-resistant tumor model. It has been reported that treatment with $30-60 \mathrm{mg} / \mathrm{kg}$ of sorafenib led to complete tumor stasis in the A549 xenograft (Wilhelm et al., 2004). Therefore, in this study, A549 tumor-bearing athymic nude mice were given oral administration of $40 \mathrm{mg} / \mathrm{kg}$ per day of sorafenib. The treatment was well tolerated by the animals. No deaths nor obvious side effects were observed. At such dose level, a highly statistically significant inhibition of tumor growth corresponding to a mean tumor size reduction of up to $86 \%$ was observed. Mice in the control group reached the predetermined endpoint (tumor volume $=1000 \mathrm{~mm}^{3}$ ) earlier and thus were in the study for a shorter period than were the sorafenib-treated mice ( 6 weeks for mice in the control group versus 15 weeks for those in the sorafenib-treated group) (Fig. 1A). During the 15-week treatment period, sorafenib-treated mice exhibited different tumor growth rates. The fold change of tumor volume (i.e., the tumor growth index value) after the 15 -week treatment period ranged from 0.9 to 30.2 with the median value of 3.6 (Fig. 1B). The fastest growing sorafenibtreated A549 xenograft with a tumor growth index value of 30.2 was used to derive the A549/SRFres subclone for the subsequent study. Further evaluation of the A549/SRFres xenograft model compared with the A549 xenograft model was conducted in individual mice receiving simultaneous subcutaneous inoculations of A549 cells on the left flank and A549/SRFres cells on the right flank (Fig. 1C). Two of the vehicle-treated animals and two of the sorafenib-treated animals were euthanized after 8 weeks of treatment, and one vehicle-treated animal was euthanized after 10 weeks of treatment because their tumors reached the maximum allowed volume (i.e., $1000 \mathrm{~mm}^{3}$ ). One time-matched sorafenib-treated animal was euthanized after 10 weeks of treatment. No significant difference was seen in tumor volume among all study groups when the treatment was initiated 1 week after tumor inoculation (Supplemental Fig. 1). Significant suppression of tumor growth by sorafenib treatment was observed from weeks $9-12(P<0.05$ for both A549 and A549/SRFres tumors compared with the corresponding vehicle control (Fig. 1D). A549/SRFres tumors exhibited relatively rapid tumor growth rates compared with A549 tumors during the 12-week treatment period. For the vehicle control group, the difference in tumor volume between A549 and A549/SRFres tumors was statistically significant at weeks 2 , $3,4,5,6$, and $12(P<0.05$, Fig. 1D). For the sorafenib treatment group, the significant difference in tumor volume between A549/SRFres tumors and their parental counterparts was observed at weeks $2,3,5,6,9,10$, and $11(P<0.05$. Fig. 1D). The mean tumor growth index value for the A549/SRFres xenografts was higher than that for the A549 xenografts (13.7 \pm 3.9 vs. $8.7 \pm 5.3$ ), but the difference was not statistically significant $(P=0.184)$. In terms of tumor response to sorafenib treatment, sorafenib effectively suppressed the growth of both A549 and A549/SRFres xenografts as demonstrated by the $67 \%(P<0.05)$ and $56 \%(P<0.01)$ decrease in the mean tumor growth index values for A549 and A549/SRFres tumors, respectively. Comparison of tumor growth rate of the sorafenib-treated A549/SRFres tumors and their parental counterparts showed that the mean volumes of A549/SRFres tumors were significantly larger than those of the A549 tumors at weeks 3,4 , and 6 after the start of the treatment $(P<0.05)$. Moreover, the mean tumor growth index value for the sorafenib-treated A549/SRFres tumors was significantly greater than that of the A549 tumors (6.0 23.9 vs. $2.9 \pm 1.3$, $P<0.05)$. These results suggest that the A549/SRFres tumors display relatively greater resistance to sorafenib than their parental counterparts. Taken together, sorafenib-treated A549/SRFres tumors demonstrated significantly rapid growth compared with their sorafenib-treated parental counterparts, which may be attributable in part to the relatively rapid baseline A549/SRFres tumor growth.

Identification of Potential Mechanisms Associated with Acquired Sorafenib Resistance. To identify the molecular mechanisms involved in the acquired sorafenib resistance, Western blot analysis was performed to assess the levels of selected epithelial-mesenchymal transition (EMT)

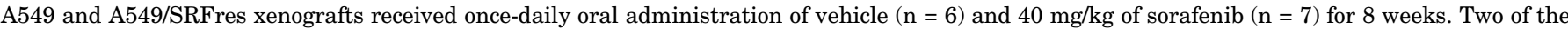

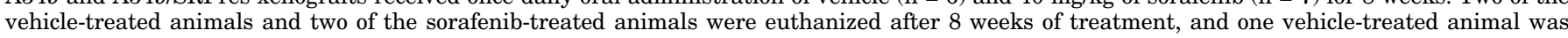

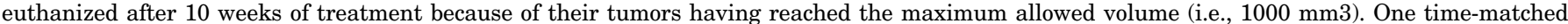

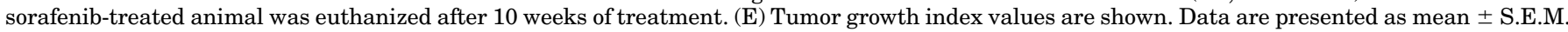

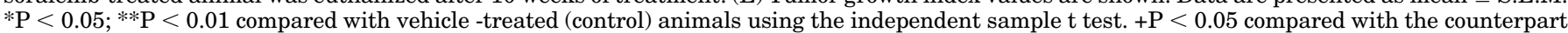
A549 xenografts using the paired sample t test. 
markers and protein kinases in vehicle- and sorafenib-treated A549 and A549/SRFres tumors (Fig. 2, A and B). For the A549 xenografts, a significant decrease in the $\beta$-catenin expression level was found in sorafenib-treated tumors compared with the control tumors (44\% decrease, $P<0.05$. Fig. 2B). With regard to the A549/SRFres xenografts, both $\beta$-catenin and E-cadherin expression levels were significantly decreased (36\% decrease and $P<0.05$ for both), whereas the MMP9 (362\% increase, $P<0.01$ ) and phospho-FAK (138\% increase, $P<0.05$. Fig. 2B) levels were significantly increased in the sorafenib-treated tumors compared with those in the control tumors. Expression of phospho-Src in the A549 and A549/SRFres tumors showed 56\% and 59\% increases, respectively, although statistical significance was not reached $(P=0.099$ and 0.069 for A549 and A549/SRFres tumors, respectively). Nonetheless, given the prominent role of Src in the receptor tyrosine kinase (RTK)-mediated signal transduction through the activation of RAS/RAF/MAPK and PI3K/AKT pathways that promotes cell survival, mitogenesis, and migration and invasion (Bromann et al., 2004; Thamilselvan et al., 2007), this finding prompted us to speculate that activation of FAK-Src signaling pathway creates a bypass track that promotes resistance to sorafenib, and the use of a Src inhibitor in combination with sorafenib may antagonize sorafenib resistance conferred by the FAKSrc-mediated bypass signaling pathway.
A
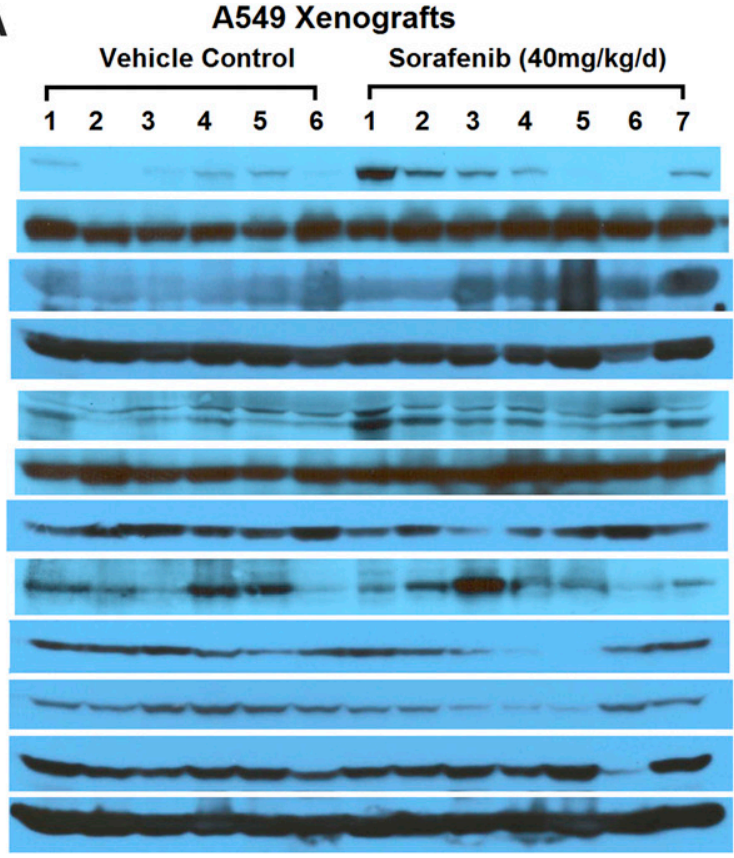

B

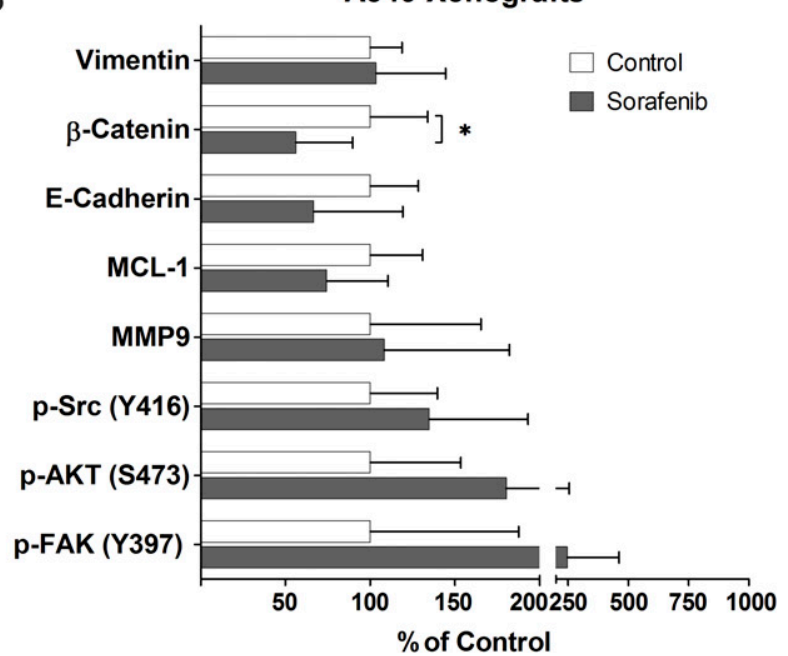

A549/SRFres Xenografts

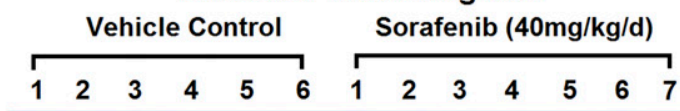

P-FAK (Y397)

FAK

p-AKT (S473)

AKT

p-Src (Y416)

SRC

MCL-1

MMP9

E-Cadherin

$\beta$-Catenin

Vimentin

$\beta$-Actin
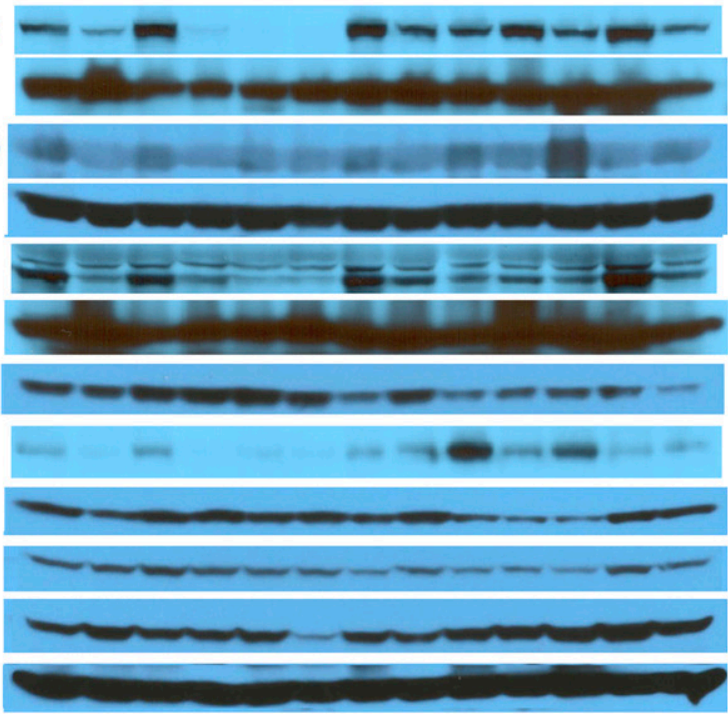

A549/SRFres Xenografts

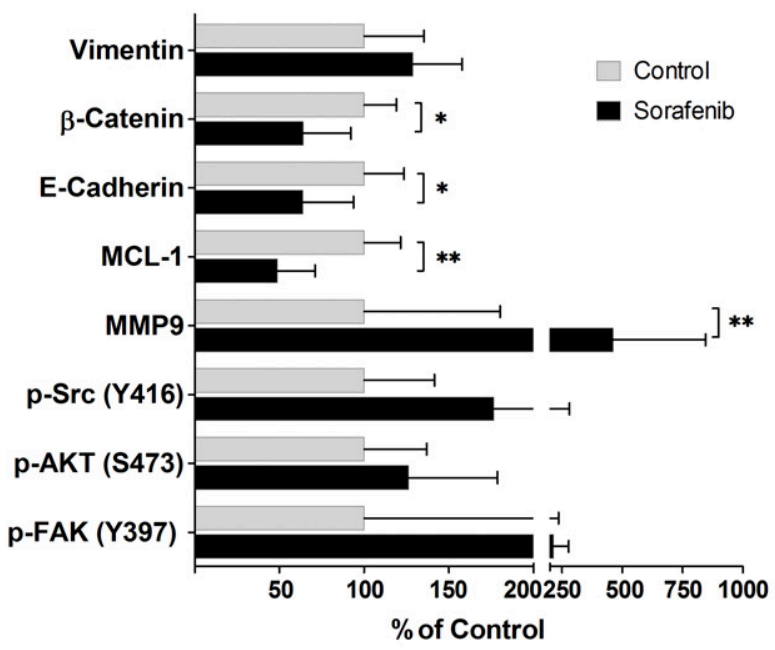

Fig 2 Western blot analysis showing that sorafenib induces MMP9 expression and phosphorylation of FAK, Src, and AKT and decreases the expression of E-cadherin (the epithelial marker) and $\beta$-catenin in A549 and A549/SRFres xenografts. (A) Western blot of A549 (left) and A549/SRFres (right) tumor homogenates to detect the proteins indicated. (B) Densitometric analysis shows similar trends in the effect of sorafenib on individual protein expression levels in A549 (left) and A549/SRFres (right) tumors. Relative quantification is performed, and relative immunoreactive band intensities are expressed as percent change over the average signal value in vehicle control tumors with normalization to $\beta$-actin-loading controls. Error bars are S.D. *P $<0.05$; $* * \mathrm{P}<0.01$ compared with vehicle-treated (control) xenografts using the independent sample t test. 
No Changes in Proliferation and Migratory and Invasive Phenotype in A549/SRFres Cells Compared with A549 Parental Cells In Vitro. To examine the impact of long-term in vivo therapeutic pressure imposed by sorafenib on the in vitro behavior of A549/SRFres cells, measurement of doubling time, wound-healing assay, and transwell invasion assay were conducted. No significant difference in doubling time was found between A549 parental and A549/SRFres cells ( $23.7 \pm 1.9$ hours vs. $24.3 \pm 1.7$ hours, $P>0.05$ ). Moreover, the migratory (Fig. 3A) and invasive (Fig. 3B) phenotypes of A549/ SRFres cells appeared similar to those of A549 cells $(P>0.05$ for all). These data suggest that the aggressive behavior of A549/SRFres cells acquired in vivo under the therapeutic pressure is reversible.

Examination of Molecular Changes Induced by Sorafenib and Dasatinib Treatment in Cultured A549 and A549/SRFres Cells. To determine whether activation of the FAK-Src signaling pathway was responsible for the acquired sorafenib-resistance phenotype in A549 xenograft tumors, the baseline and treatment-induced changes in protein expression levels of EMT markers and downstream signaling molecules regulating cell proliferation, migration, and invasion were compared in A549 parental and A549/ SRFres cells. As shown in Fig. 3C, the baseline expression levels of phospho-Src (Y416) and phospho-FAK (Y576/577) were markedly increased by $60 \%$ and $110 \%$, respectively, in A549/SRFres cells compared with those in A549 parental cells. Treatment with $10 \mu \mathrm{M}$ sorafenib for 4 hours had no effect on the expression of phospho-Src and phospho-FAK (Y576/577). In contrast, dasatinib treatment at 0.02 and $0.2 \mu \mathrm{M}$ was sufficient to inhibit the phosphorylation of Src at Tyr-416 and FAK at Tyr-576/577. Moreover, the combined sorafenib and dasatinib treatment appeared to result in a greater decrease in Src phosphorylation in A549/SRFres cells than it did in A549 cells, suggesting that A549/SRFres cells with elevated phospho-Src levels are relatively sensitive to the sorafenibdasatinib combination treatment. No notable difference was found in either baseline expression levels or treatmentinduced changes in the expression levels of other proteins evaluated in A549 parental and A549/SRFres cells.

In Vitro Evaluation of Cytotoxic Effect of SorafenibDasatinib Combination in A549 Parental and A549/ SRFres Cells. To evaluate the in vitro cytotoxic effect of the combination treatment with sorafenib and dasatinib in A549 and A549/SRFres cells, sorafenib and dasatinib were used at the fixed molar ratios of 1:3, 1:1, and 3:1. Quantitative analyses of the dose-effect relationships of sorafenib and dasatinib treatment alone and in combination showed that the $\mathrm{IC}_{50}$ values for sorafenib and dasatinib alone in the A549 cell line were similar to those in its sorafenib-resistant subclone (i.e., A549/SRFres), whereas the combined sorafenib and dasatinib treatment resulted in a greater inhibitory effect on cell proliferation than treatment with either drug alone in both cell lines (Fig. 4A). In applying the CI method to the data in Fig. 4A, we assumed that sorafenib and dasatinib were mutually nonexclusive; that is, the action of sorafenib on RAF/ VEGFR /PDGFR did not affect the action of dasatinib on Src/ ABL. For A549/SRFres cells, simultaneous and continuous exposure to sorafenib and dasatinib at the fixed molar ratios of 1:1 and 3:1 for 72 hours showed synergism $(\mathrm{CI}<1)$ for the fraction affected (FA) values between 0.1 and 0.9 , whereas the combination of sorafenib and dasatinib at the fixed molar ratio of 1:3 showed synergism $(\mathrm{CI}<1)$ when FA values were greater than 0.4. For A549 cells, simultaneous exposure to sorafenib and dasatinib at the molar ratio of 1:1 produced an additive to weak synergistic effect for FA values ranging from 0.2 to 0.8 , whereas sorafenib and dasatinib at the molar ratio of $1: 3$ and 3:1 resulted in an additive to synergistic effect for $F A$ values greater than 0.4 (Fig. 4B; Supplemental Table 1). Taken together, these data clearly indicate that combination treatment with sorafenib and dasatinib is effective against A549 and A549/SRFres cells when used at a molar concentration ratio between $1: 3$ and 3:1.

In Vivo Response of A549 and A549/SRFres Xenograft Models to Sorafenib and Dasatinib Alone and in Combination. Since we observed the additive and synergistic cytotoxic effects of concurrent treatment with sorafenib and dasatinib on A549 and A549/SRFres cells in vitro, we next evaluated the antitumor effect of individual and combination treatments with sorafenib and dasatinib on the established A549 and A549/SRFres lung cancer xenografts. No significant difference in tumor volume was observed among all study groups when the treatment was initiated 3 weeks after tumor inoculation (Supplemental Figs. 2 and 3). Sorafenib and dasatinib monotherapies at the dose of $20 \mathrm{mg} / \mathrm{kg}$ per day significantly suppressed the growth of A549/SRFres xenografts with the maximum percent tumor growth inhibition of $45 \%(P<0.01)$ and $35 \%(P<0.05)$, respectively, but were unable to significantly inhibit the growth of A549 tumors (Supplemental Fig. 3). As shown in Fig. 4C, in A549 xenografts, the effect of $40 \mathrm{mg} / \mathrm{kg}$ per day sorafenib on tumor growth arrest became significant 1.5 weeks after the start of the treatment with a maximum percent tumor growth inhibition of $72 \%(P<0.01$ compared with controls). Tumor growth inhibition did not become significant until 3.5 weeks after the start of the sorafenib-dasatinib combination treatment; maximum percent tumor growth inhibition was 53\% $(P<0.05$. Fig. 4C). Only $30 \%$ maximum tumor growth inhibition was achieved with $40 \mathrm{mg} / \mathrm{kg}$ per day dasatinib treatment alone $(P>0.05$. Fig. $4 \mathrm{C})$. In A549/SRFres xenografts, the inhibitory effect of sorafenib alone and half-dose combination of sorafenib and dasatinib on tumor growth became significant 1 week after start of the treatment; the maximum percent tumor growth inhibition was $75 \%$ and $69 \%$, respectively $(P<0.01$ compared with controls for both. Fig. 4D). In contrast, although dasatinib monotherapy was able to produce a maximum percent tumor growth inhibition of $52 \%$ ( $P>0.05$ compared with controls; Fig. 4D), the inhibitory effect of dasatinib on tumor growth was not statistically significant except for after week $3(P<0.05$. Fig. 4D). Compared with the parental counterparts, vehicle- and sorafenib-treated A549/SRFres tumors demonstrated a significantly greater tumor growth rate $(P<0.05$ or $P<0.01$ using paired sample $t$ test; Fig. 4D); however, tumor growth rates of dasatinib- and combination-treated A549/SRFres tumors did not differ significantly from those of their corresponding parental counterparts $(P>0.05$. Fig. 4D). Overall, in both A549 and A549/SRFres xenografts, treatment with sorafenib alone at $40 \mathrm{mg} / \mathrm{kg}$ per day resulted in the most significant tumor growth inhibition, which was significantly more than that achieved by treatment with $40 \mathrm{mg} / \mathrm{kg}$ daily dasatinib. The half-dose sorafenib-dasatinib combination was more effective than the $20 \mathrm{mg} / \mathrm{kg}$ daily dasatinib monotherapy in both A549 $(P<0.05)$ and A549/SRFres $(P<0.01)$ 
A
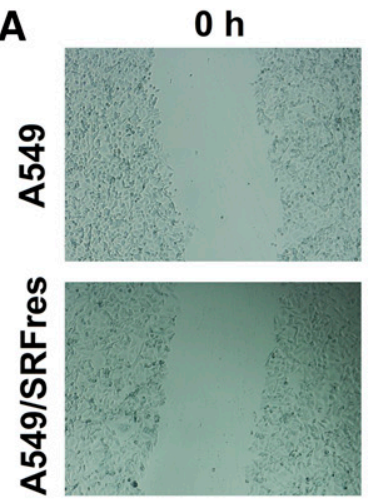

B

C

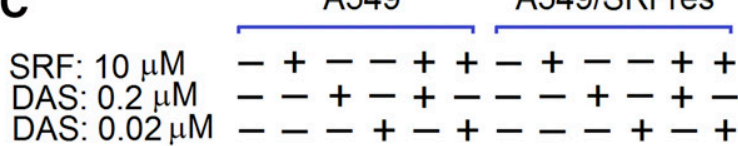

FAK

p-FAK(Y397)
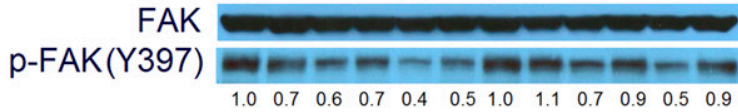

p-FAK(Y576)

$\begin{array}{llllllllllll}1.0 & 0.9 & 0.0 & 0.6 & 0.0 & 0.3 & 2.1 & 2.2 & 0.2 & 0.6 & 0.0 & 0.1\end{array}$

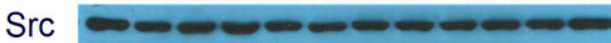

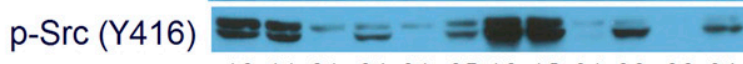

$\begin{array}{llllllllllll}1.0 & 1.1 & 0.1 & 0.4 & 0.1 & 0.7 & 1.6 & 1.5 & 0.1 & 0.6 & 0.0 & 0.4\end{array}$

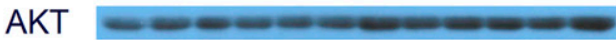

p-AKT (S473)
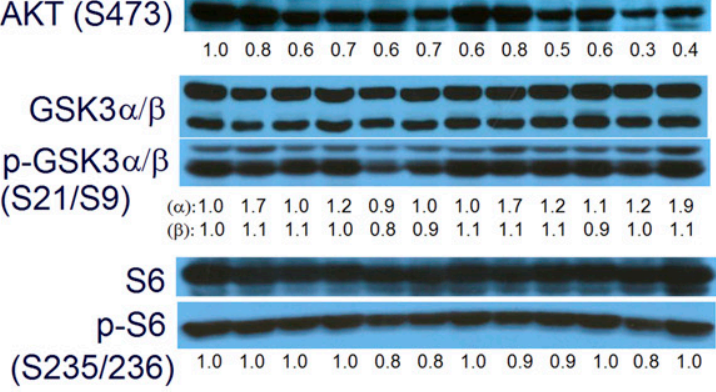

$48 \mathrm{~h}$

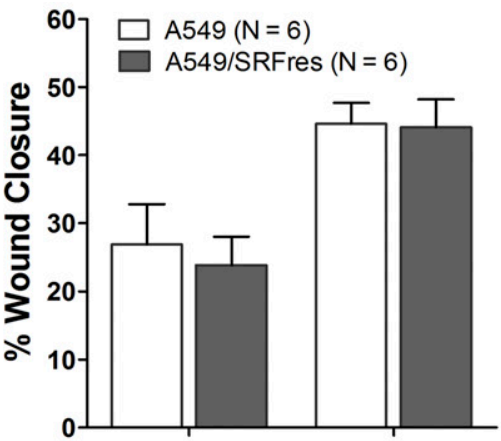

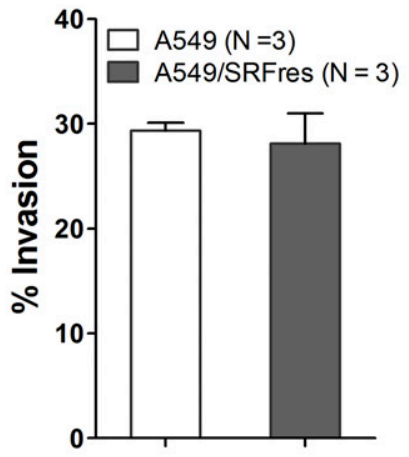

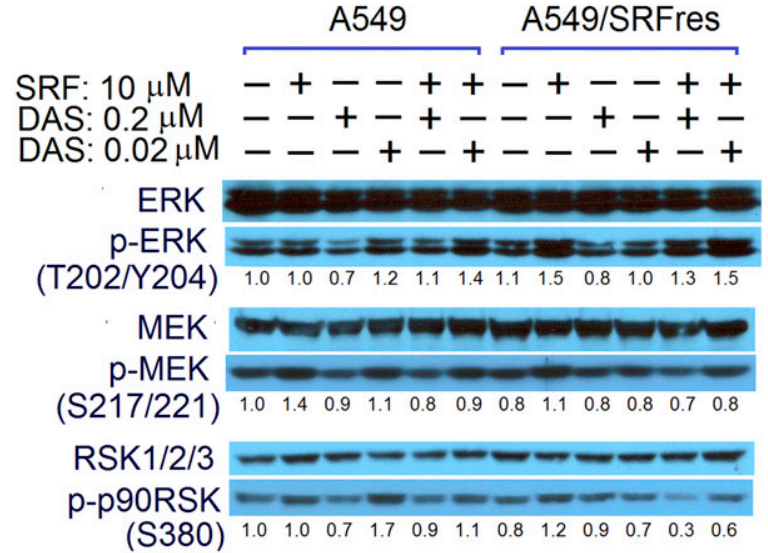

MCL-1

\section{E-Cadherin \\ $\begin{array}{llllllllllll}1.0 & 1.1 & 1.0 & 0.8 & 0.6 & 0.7 & 0.9 & 1.2 & 1.0 & 0.8 & 0.9 & 0.9\end{array}$}

$\begin{array}{llllllllllll}1.0 & 0.8 & 0.8 & 0.7 & 0.2 & 0.3 & 0.9 & 0.7 & 0.8 & 0.9 & 0.5 & 0.6\end{array}$

\section{$\beta-$ Catenin}

Vimentin
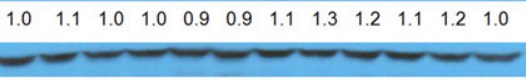

$\begin{array}{llllllllllll}1.0 & 1.0 & 0.9 & 0.8 & 0.9 & 0.9 & 1.1 & 1.2 & 1.2 & 1.2 & 1.1 & 0.7\end{array}$

$\alpha-S M A$

$\begin{array}{llllllllllll}1.0 & 1.3 & 1.1 & 1.0 & 0.9 & 1.0 & 1.1 & 1.4 & 1.2 & 1.4 & 1.0 & 1.1\end{array}$

$\beta$-Actin

Fig 3 No significant difference was found in the migratory and invasive phenotypes of A549 parental and A549/SRFres cells; however, differential expression of phospho-Src (Y416) and phospho-FAK (Y576/577) were observed between those two cell lines. (A) Representative micrographs of scratchwound closure kinetics of cultured A549 and A549/SRFres cells. Quantification of relative wound area at 24 and 48 hours after wounding of the cell monolayers, expressed as the percentage of wound closure, demonstrated no significant difference in the migratory ability between those two cell lines in vitro. Error bars represent the S.D. of the mean from six independent experiments. (B) Representative micrographs from the transwell invasion assay performed by plating A549 and A549/SRFres cells on uncoated and Matrigel-coated transwell membranes. No significant difference was noted in the in vitro invasion ability between those two cells based on the percent invasion value, which was calculated as the percentage of invaded cells relative to 
xenografts, and it was also more effective than the $20 \mathrm{mg} / \mathrm{kg}$ per day sorafenib treatment in A549/SRFres xenografts $(P<$ 0.05. Supplemental Fig. 4). No significant difference in mean tumor volumes was observed between the $40 \mathrm{mg} / \mathrm{kg}$ per day sorafenib monotherapy and half-dose sorafenib-dasatinib combination groups in either A549 or A549/SRFres xenografts $(P>0.05$. Fig. 4, C and D). Moreover, significantly rapid tumor growth was observed in vehicle- and sorafenib-treated A549/ SRFres tumors, but not in dasatinib- and combination-treated A549/SRFres tumors, compared with their corresponding parental counterparts.

Effects of Sorafenib and Dasatinib Alone and in Combination on Tumor Angiogenesis, Tumor Cell Proliferation, and Adhesion in A549 and A549/SRFres Xenografts. Results of immunofluorescence staining of cultured A549 and A549/SRFres cells demonstrated that there was no difference in baseline Ki67, E-cadherin, $\alpha$-SMA, and collagen IV expression levels between those two cell lines (Supplemental Fig. 5). Nonetheless, the observed tumor growth inhibition after treatment with full-dose sorafenib and half-dose sorafenib-dasatinib combination in the A549 and A549/SRFres xenograft models warranted further assessment of the mechanisms underlying the in vivo antitumor activities of individual and combination treatments. Proliferation is a key feature of tumor progression and is often estimated by the immunohistochemical assessment of the nuclear antigen Ki67. Results from this study demonstrated a decrease in the mean Ki67 proliferative index values after the full-dose sorafenib and half-dose combination therapies in both A549 and A549/SRFres xenografts, although only the decrease in A549/SRFres tumors was statistically significant compared with the control tumors $(P<0.05$ for both full-dose sorafenib and half-dose combination therapies; Fig. 5, A and B). Nonetheless, the mean Ki67 proliferative index value in sorafenib-treated A549/SRFres tumors was significantly increased compared with that in their parental counterparts (by $38 \%, P<0.05$ ), suggesting an attenuated sorafenib antiproliferative activity in A549/SRFres tumors (Fig. 5B). In addition, the Ki-67 proliferative index values were highly correlated with the volumes of both A549 and A549/SRFres tumors at week 4 (Pearson: $r=0.544$ for A549, $r=0.542$ for A549/SRFres, $n=28$ and $P<0.01$ for both), suggesting that inhibitory effect of sorafenib and dasatinib on tumor growth is attributable to the antiproliferative activities of individual drugs. In addition, the results of Ki-67 immunofluorescence staining were in line with the results of the H\&E staining of tumor sections, which revealed that relatively large regions of necrosis were present in tumors receiving drug treatments compared with control tumors (Supplemental Fig. 6).

E-cadherin plays a crucial role in maintaining cell-cell adhesion in epithelial tissues. Downregulation of E-cadherin expression is associated with tumor progression, invasion, and metastasis (Rodriguez et al., 2012). Results of the immunofluorescence staining of tumor sections showed that E-cadherin expression levels were significantly reduced in sorafenib-treated A549 tumors (decreased by 30\%, $P<0.05$ ) but not in sorafenib treated A549/SRFres tumors (decreased by $8 \%, P>0.05)$ compared with the corresponding controls (Fig. 5C).

Effects of individual and combination treatments on tumor vasculature were evaluated using the immunohistochemical markers for endothelial cells (with CD31), microvessel basement membrane (with collagen IV), and mural cells (i.e., pericytes and smooth muscle cells with $\alpha$-SMA) (Fig. 5, A and D-F). As a potent antiangiogenic agent, sorafenib was able to reduce the microvessel density (MVD) determined by the CD31 positive staining in both A549 $(P<0.01)$ and A549/ SRFres $(P<0.05)$ xenografts, whereas full-dose dasatinib treatment resulted in a significant decrease in MVD in A549 xenografts $(P<0.05)$, but not in A549/SRFres xenografts. Notably, the mean MVD in dasatinib-treated A549/SRFres tumors was significantly higher than that in their parental counterparts $(P<0.05)$, suggesting that dasatinib has no effect on tumor angiogenesis in A549/SRFres tumors. A significant decrease in MVD after the half-dose sorafenibdasatinib combination therapy was seen in A549/SRFres tumors $(P<0.05)$ but not A549 tumors (Fig. 5D). Full-dose sorafenib treatment significantly reduced collagen IV expression levels in A549 tumors, but not in A549/SRFres tumors, compared with the vehicle controls and half-dose sorafenibdasatinib combination $(P<0.05$ for both). Full-dose dasatinib treatment significantly increased collagen IV expression in A549/SRFres tumors compared with that in their parental counterparts $(P<0.05)$, which was consistent with its diminished effect on MVD in A549/SRFres tumors. Compared with full-dose dasatinib treatment, the half-dose combination therapy was able to lower the collagen IV expression levels in A549/SRFres tumors significantly $(P<0.05)$ (Fig. 5E). As for $\alpha$-SMA, no statistically significant difference was found among the study groups, suggesting that none of the treatments affects mural cells in the tumor (Fig. 5F). Taken together, the results of the immunofluorescence staining of tumor tissues confirmed that the antitumor effect of sorafenib and dasatinib was attributable to their antiproliferative and antiangiogenic properties, and A549/SRFres tumors exhibited a relatively higher cell proliferation rate than A549 tumors.

Mechanisms Underlying the Antitumor Effects of Sorafenib and Dasatinib Alone and in Combination in A549 and A549/SRFres Xenografts. In addition to the histologic analyses, Western blot analysis was carried out to elucidate the molecular events underpinning the tumor growth inhibition mediated by sorafenib and dasatinib alone and in combination and identify any potential escape routes developed during the treatment (Supplemental Figs. 7 and 8). In A549 xenografts, compared with the control tumors, the $40 \mathrm{mg} / \mathrm{kg}$ per day sorafenib treatment significantly decreased expression of MCL-1 (by 38\%, $P<0.01$ ) and phospho-GSK3 $\alpha$ (by $55 \%, P<0.01$ ) but increased expression of phospho-AKT (by $30 \%, P<0.05$ ). The $40 \mathrm{mg} / \mathrm{kg}$ per day dasatinib treatment, on the other hand, significantly decreased the expression of

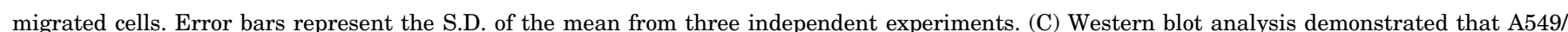

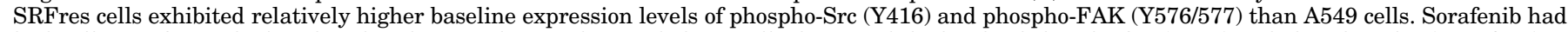

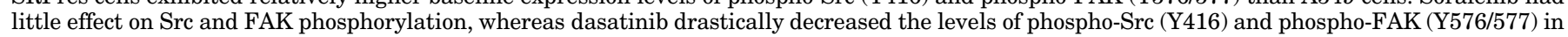

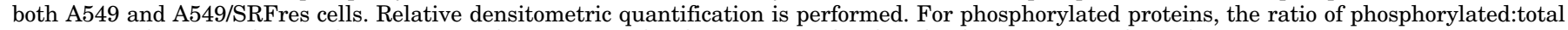
species was determined. For other proteins, relative protein levels were normalized to the densitometry values of $\beta$-actin. 

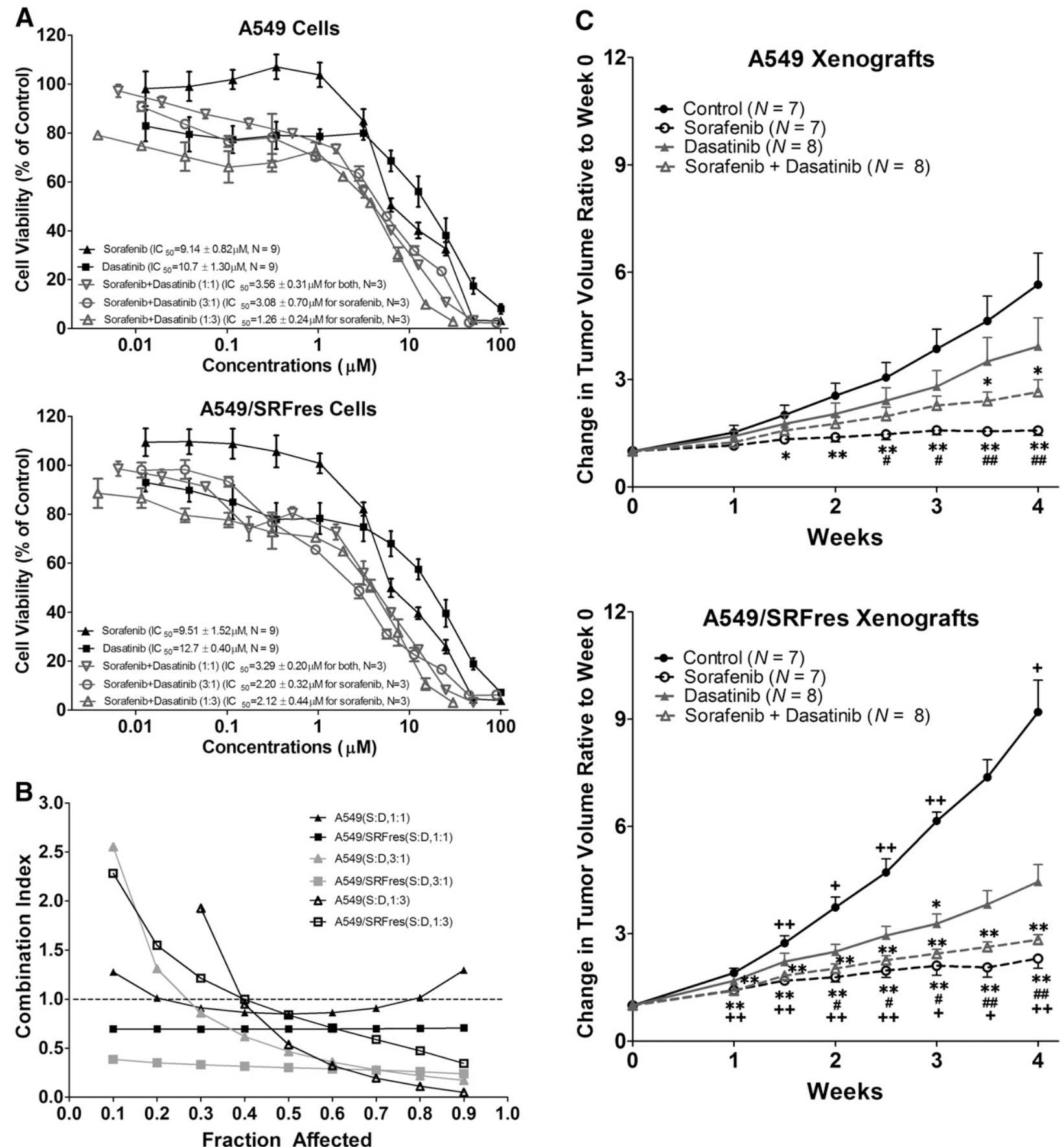

Fig 4 Sorafenib and dasatinib, alone and in combination, inhibit A549 and A549/SRFres cell growth in vitro and in vivo. (A) Composite dose-effect curves for in vitro antiproliferative activity of sorafenib and dasatinib alone $(0-100 \mu \mathrm{M})$ and in combination (at fixed molar ratios of sorafenib/dasatinib $=$ 1:3, 1:1, and 3:1) in A549 (top left) and A549/SRFres (middle left) cells. Error bars are interassay S.D. (B) The corresponding combination index for the sorafenib-dasatinib interaction in A549 and A549/SRFres cells as a function of fraction affected. (C) Antitumor activity of sorafenib and dasatinib, as single agents or in combination, in A549 (top right) and A549/SRFres (bottom right) xenografts. Mice bearing subcutaneous A549 and A549/SRFres tumors were treated with oral administration of $40 \mathrm{mg} / \mathrm{kg}$ of sorafenib, $40 \mathrm{mg} / \mathrm{kg}$ of dasatinib, or the combination of the two agents at half doses for 4 weeks. Values indicate mean \pm S.E.M. $* \mathrm{P}<0.05$; $* * \mathrm{P}<0.01$ compared with the vehicle treated (control) animals. \#P $<0.05$; \#\#P $<0.01$ compared with the dasatinib-treated animals using Kruskal-Wallis one-way analysis of variance on ranks, followed by the post hoc Kruskal-Wallis multiple comparison z-value test. $+\mathrm{P}<0.05 ;++\mathrm{P}<0.01$ compared with the counterpart $\mathrm{A} 549$ xenografts using the paired sample $t$ test.

phospho-Src (by $50 \%, P<0.05$ ) and phospho-ERK (by $19 \%$, $P<0.05$ ) but increased the Y397-FAK phosphorylation (by $191 \%, P<0.05)$. Half-dose sorafenib-dasatinib combination resulted in a significant decrease in the expression of phosphoERK (by $21 \%, P<0.05$ ) and a significant increase in the Y397FAK phosphorylation (by $119 \%, P<0.05$ ). In addition, expression of phospho-Src in $40 \mathrm{mg} / \mathrm{kg}$ daily dasatinibtreated A549 tumors was 58\% lower than that in sorafenibtreated A549 tumors $(P<0.05)$, whereas the phospho-AKT expression in A549 tumors treated with the half-dose combination was $26 \%$ lower than that in sorafenib-treated A549 tumors $(P<0.05)$ (Fig. 6A). In similar fashion, $20 \mathrm{mg} / \mathrm{kg}$ per 
A

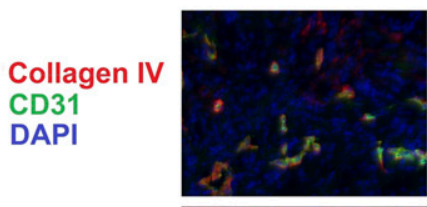

\section{E-Cadherin \\ CD31 \\ DAPI}

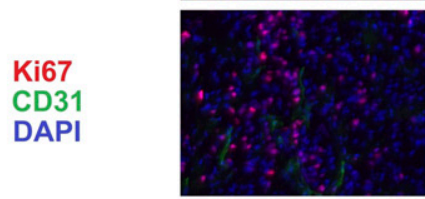

$\alpha$-SMA

CD31

DAPI
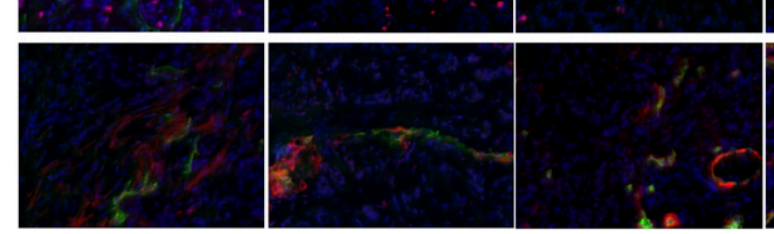

\section{Collagen IV \\ CD31 \\ DAPI}
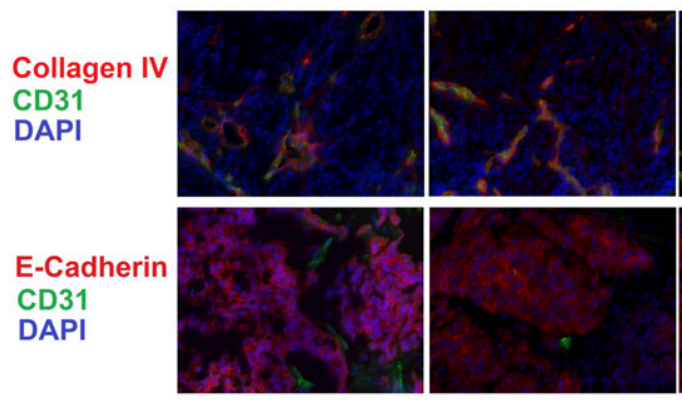

Ki67

CD31

DAPI
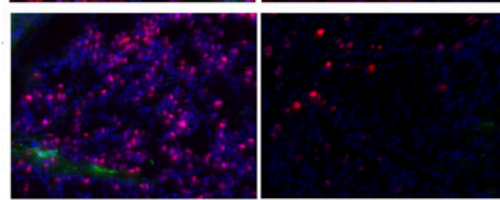

$\alpha$-SMA

CD31

DAPI

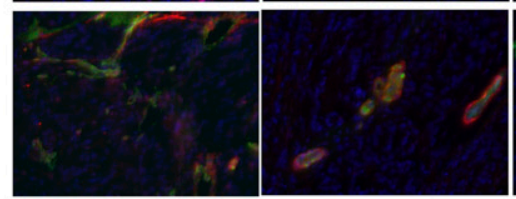

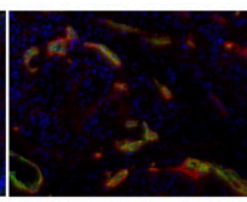
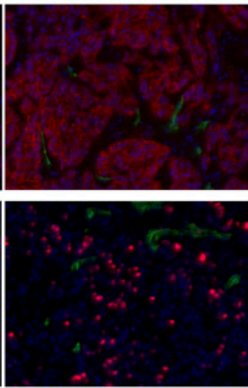

Dasatinib
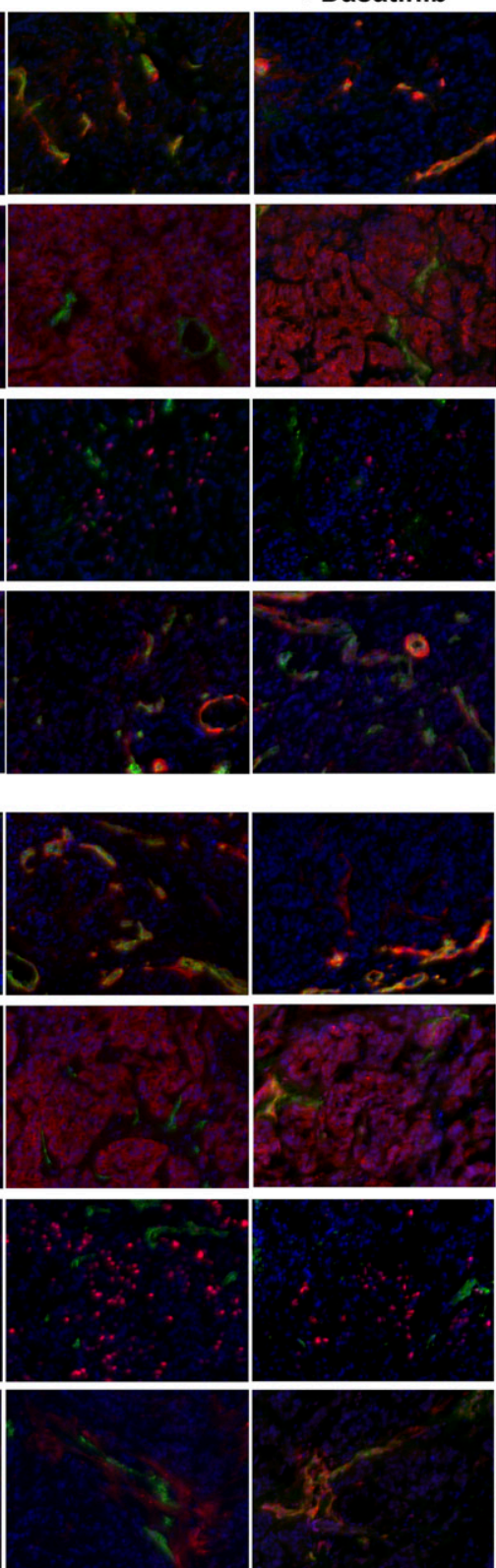

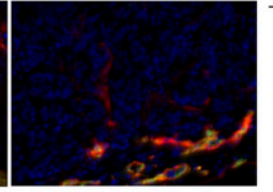

Sorafenib

+ Dasatinib
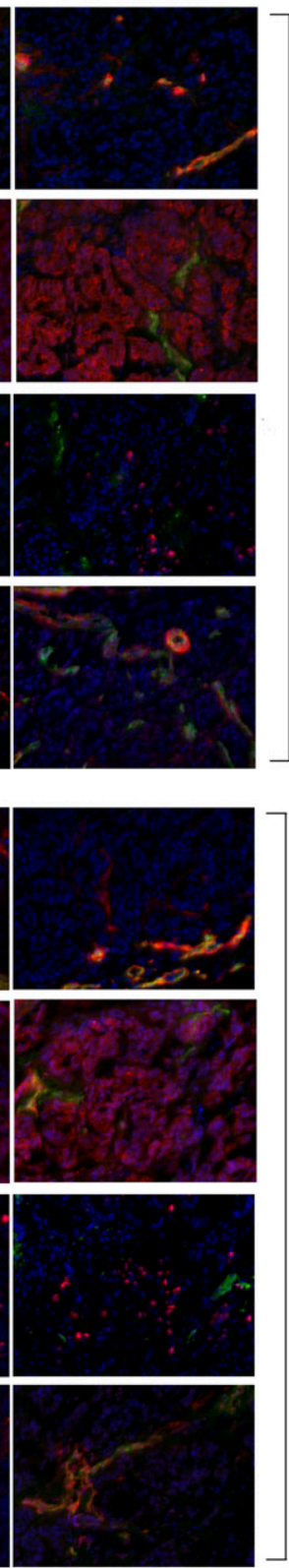
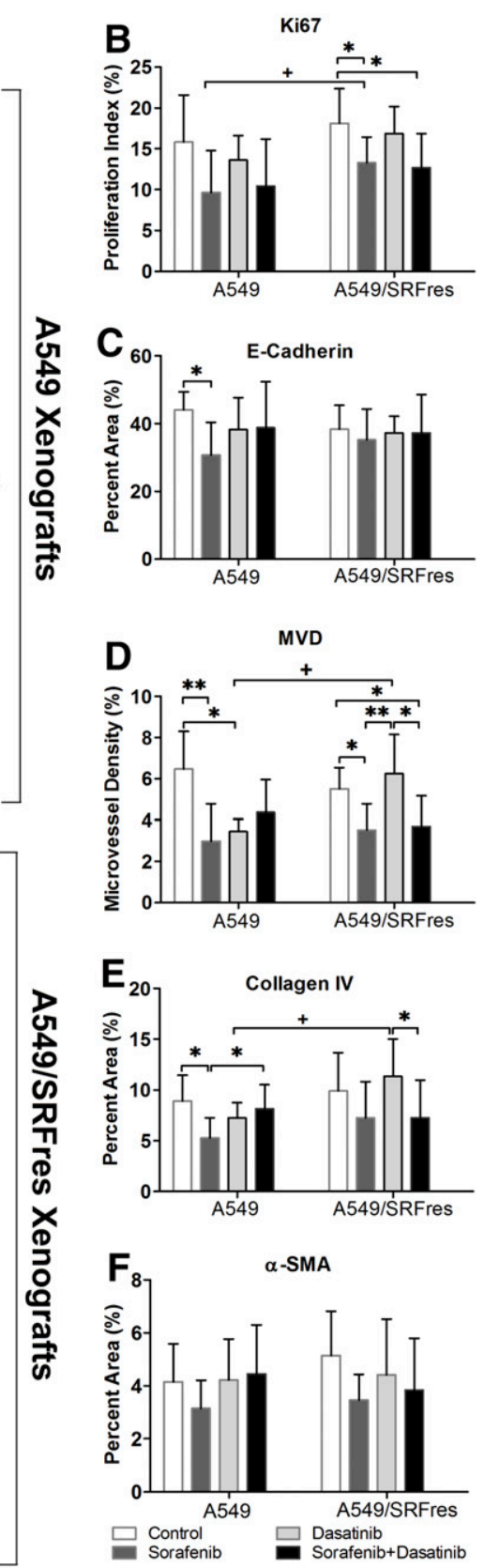

Fig 5 Immunohistochemical analyses of A549 and A549/SRFres xenografts. Tumors were harvested from individual mice bearing both A549 and A549/ SRFres xenografts after 28 days of treatment with vehicle control $(\mathrm{n}=6)$, sorafenib alone $(40 \mathrm{mg} / \mathrm{kg}$ per day, $\mathrm{n}=7)$, or dasatinib alone ( $40 \mathrm{mg} / \mathrm{kg}$ per day, $\mathrm{n}=7$ ), or sorafenib in combination with dasatinib $(20 \mathrm{mg} / \mathrm{kg}$ per day for each, $\mathrm{n}=8)$. (A) Representative images for immunofluorescence double staining for CD31 (green), plus Ki67 (red), E-cadherin (red), collagen IV (red), or $\alpha$-SMA (red) in tumor sections from individual study groups. The nuclei were stained with DAPI. Original magnification, 200×. (B) Quantification of Ki67 immunofluorescence staining shown by the percentage of Ki67-positive cells compared with total number of cells per field. (C) Quantification of E-cadherin immunofluorescence staining shown by the percentage of E-cadherin positive area compared with the tumor area per field. (D) Quantification of immunofluorescence staining for microvessel baseline membrane shown by the percentage of collagen IV-stained area per field. (E) Quantification of collagen IV immunofluorescence staining for mural cells shown by the percentage of $\alpha$-SMA-stained area per field. (F) Quantification of immunofluorescence staining for MVD shown by the percentage of CD31-stained area per field. Error bars are S.D. *P $<0.05 ; * * \mathrm{P}<0.01$ using Kruskal-Wallis one-way analysis of variance on ranks followed by the post hoc Kruskal-Wallis multiple comparison $\mathrm{z}$-value test. $+\mathrm{P}<0.05$ using paired-sample $\mathrm{t}$ test.

day of sorafenib treatment significantly reduced the phosphorylation of GSK3 $\alpha(P<0.05)$, whereas $20 \mathrm{mg} / \mathrm{kg}$ per day dasatinib treatment resulted in significant decrease in Y416Src phosphorylation and Y576/577-FAK phosphorylation $(P<0.05$ and $P<0.01$ compared with vehicle and treatment with sorafenib, $20 \mathrm{mg} / \mathrm{kg}$ per day, respectively; Supplemental Fig. 8A). In A549/SRFres tumors, compared with control tumors, sorafenib (40 mg/kg per day) significantly decreased the expression of MCL-1 and phospho-GSK3 $\alpha$ by $16 \%$ and $53 \%$, respectively $(P<0.05$ for both), but it increased the phospho-Src expression by $114 \%(P<0.05)$. Dasatinib treatment $(40 \mathrm{mg} / \mathrm{kg}$ per day) significantly reduced expression of phospho-GSK3 $\alpha$ and phospho-MEK by $38 \%(P<0.05)$ and $21 \%(P<0.01)$, respectively, but it increased the Y397-FAK 
phosphorylation by $177 \%(P<0.05)$ compared with controls. Moreover, the half-dose combination therapy significantly decreased the expression of MCL-1 and phospho-GSK3 $\alpha$ by $17 \%$ and $38 \%(P<0.05$ for both), respectively, whereas the Y397-FAK phosphorylation increased by $192 \%(P<0.01)$ compared with controls. Furthermore, expression of phosphoSrc in sorafenib-treated $(40 \mathrm{mg} / \mathrm{kg}$ per day) A549/SRFres tumors was significantly increased compared with that in A549/SRFres tumors treated with dasatinib alone $(40 \mathrm{mg} / \mathrm{kg}$ per day) alone (by 95\%, $P<0.01$ ) and the combination of sorafenib and dasatinib (by $71 \%, P<0.05$ ), suggesting that dasatinib effectively inhibits Src activation in tumors. In addition, the phospho-AKT expression level in the half-dose combination group was lower than that in the full-dose dasatinib group (by 17\%, $P<0.05$ ) (Fig. 6C). Similarly, $20 \mathrm{mg} / \mathrm{kg}$ per day sorafenib and dasatinib monotherapies significantly decreased the expression of phospho-GSK3 $\alpha$ $(P<0.05$ for both). Expression of phospho-Src $(P<0.05)$, phospho-AKT $(P<0.05)$, and phospho-p90RSK $(P<0.01)$ in dasatinib-treated A549/SRFres tumors ( $20 \mathrm{mg} / \mathrm{kg}$ per day) was significantly reduced compared with that in tumors treated with sorafenib, $20 \mathrm{mg} / \mathrm{kg}$ per day (Supplemental Fig. $8 \mathrm{C})$. Results of the paired-sample $t$ test comparing the matched A549 and A549/SRFres tumor samples indicated that the phospho-ERK and phospho-GSK3 $\beta$ levels were significantly decreased in the sorafenib-treated and sorafenibdasatinib-treated A549/SRFres tumors, suggesting that ERK and GSK $3 \beta$ are unlikely to be involved in the bypass mechanism of sorafenib resistance (Fig. $6 \mathrm{E}$ ). Taken together, although dasatinib alone or in combination with sorafenib significantly reduced the phospho-Src levels in A549 and A549/SRF tumors compared with those in the sorafenib-treated tumors, the phospho-FAK (Y397) levels remained elevated in all treatment groups regardless of tumor types. In terms of other downstream effectors involved in the antitumor effect of sorafenib, including MCL-1, phospho-AKT, phospho-ERK, phospho-MEK, phosphop90RSK, and phospho-GSK-3 $\alpha / \beta$ (Liu et al., 2006; Ulivi et al., 2009), the inhibitory effect of half-dose sorafenib-dasatinib combination on the expression levels of those proteins was comparable to the full-dose sorafenib or dasatinib treatment regardless of tumor types.

Several lines of evidence have suggested that sorafenib resistance is associated with the activated EMT process and increased tumor metastatic potential (Wang et al., 2014). Therefore, we examined the effects of single agent and combination treatment on the biomarkers of EMT, including E-cadherin, $\beta$-catenin and vimentin, and the selected biomarkers of tumor metastatic potential, including MMP9 and $\alpha$-SMA, in the A549 and A549/SRFres xenograft model (Fig. 6, B, D and F; Supplemental Figs. 7 and 8). In both A549 and A549/SRFres tumors, full-dose sorafenib treatment led to a significant decrease in the expression of epithelial cell marker E-cadherin (by $44 \%$ and $P<0.01$ for A549, by $38 \%$ and $P<$ 0.05 for A549/SRFres) and a significant increase in the expression of mesenchymal cell marker vimentin (by $25 \%$ and $P<0.05$ for A549, and by $45 \%$ and $P<0.05$ for A549/ SRFres) compared with controls. Also, the expression of vimentin was significantly increased in A549 (by 20\%) and A549/SRFres (by 39\%) tumors treated with the half-dose sorafenib-dasatinib combination compared with control tumors $(P<0.05$ for both $)$. Full-dose dasatinib treatment resulted in a significant increase in MMP9 expression in
A549 (by 268\%) and A549/SRFres (by 177\%) tumors compared with vehicle controls $(P<0.01$ for both), whereas MMP9 expression levels were significantly increased in A549/SRFres tumors treated with half-dose sorafenib-dasatinib combination (by $233 \%$ and $P<0.05$ compared with controls) (Fig. 6, B and D). Sorafenib treatment at the dose of $20 \mathrm{mg} / \mathrm{kg}$ resulted in a significantly decreased $\beta$-catenin expression in A549 tumors $(P<0.05)$, whereas both $20 \mathrm{mg} / \mathrm{kg}$ per day sorafenib and dasatinib monotherapies increased the expression of vimentin in A549/SRFres tumors $(P<0.05$ for both. Supplemental Fig. $8, \mathrm{~B}$ and D). In addition, expression levels of E-cadherin and $\alpha$-SMA in the full-dose dasatinib-treated A549 tumors were significantly greater than those in the full-dose sorafenibtreated A549 tumors $(P<0.05$ for E-cadherin and $P<0.01$ for $\alpha$-SMA) (Fig. 6B). Comparison between matched A549 and A549/SRFres tumors indicated that the E-cadherin level was significantly decreased in $40 \mathrm{mg} / \mathrm{kg}$ per day sorafenib-treated A549/SRFres tumors, suggesting that A549/SRFres tumor cells are more susceptible to EMT under the therapeutic pressure imposed by sorafenib in vivo. Moreover, the MMP9 level in $20 \mathrm{mg} / \mathrm{kg}$ per day sorafenib- and $40 \mathrm{mg} / \mathrm{kg}$ per day dasatinib-treated A549/SRFres tumors was significantly lower than that in their parental counterparts, implicating that dasatinib is relatively more effective at inhibiting the progression of A549/SRFres tumors compared with that of A549 tumors (Fig. 6F; Supplemental Fig. 8F). Taken together, these findings suggest that sorafenib treatment promotes EMT in A549 xenografts, and the presence of dasatinib does not suppress the sorafenib-induced EMT, but it may increase the metastatic potential as demonstrated by the increased MMP9 and $\alpha$-SMA expression levels in tumors.

There were discrepancies between the two in vivo studies regarding the expression levels of individual EMT markers in sorafenib-treated tumors relative to those in the control tumors. For example, although sorafenib treatment resulted in significant downregulation of $\beta$-catenin and modest upregulation of vimentin in both A549 and A549/SRFres tumors in the initial study, the second in vivo study showed little change in $\beta$-catenin expression but significant upregulation of vimentin in sorafenib-treated tumors as compared with control tumors. Those discrepancies may be explained partly by the difference in time to initiate treatment ( 1 week vs. 3 weeks after tumor inoculation) and treatment duration (8 12 weeks vs. 4 weeks)

Evaluation of Sorafenib and Dasatinib Steady-State Concentrations in Plasma and Tumors. Concentrations of sorafenib and dasatinib in plasma and tumors collected at 4 hours after the last dose were quantified to determine whether the reduced effectiveness was attributable to decreased drug distribution in sorafenib-resistant tumors and if there was any pharmacokinetic drug-drug interaction in which one drug altered the tumor distribution of the other coadministered drug. As shown in Table 1, no significant difference was noted in steady-state tumor concentrations of sorafenib and dasatinib in A549 and A549/SRFres xenografts. Both sorafenib and dasatinib exhibited dose-proportional changes in plasma and tumor drug concentrations at 4 hours after the last dose, suggesting linear pharmacokinetics. Dasatinib plasma concentrations were lower than sorafenib plasma concentrations when these two drugs were given at the same dose, suggesting that dasatinib has a shorter elimination half-life than sorafenib. As a result, dasatinib tumor concentrations at 4 hours were about 


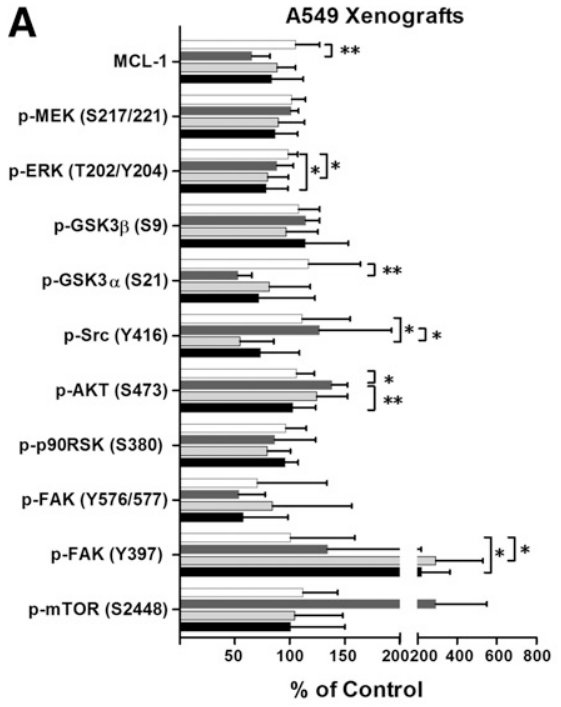

B

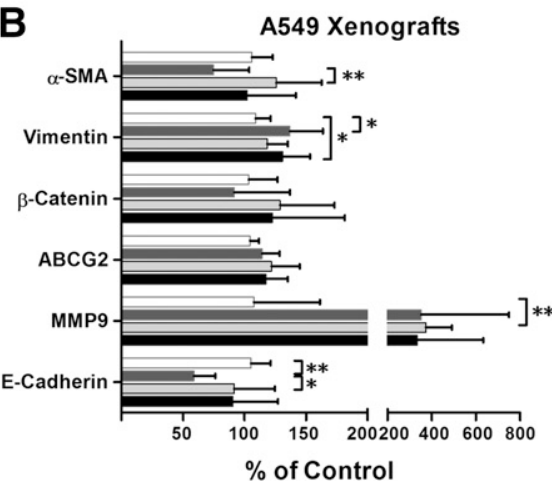

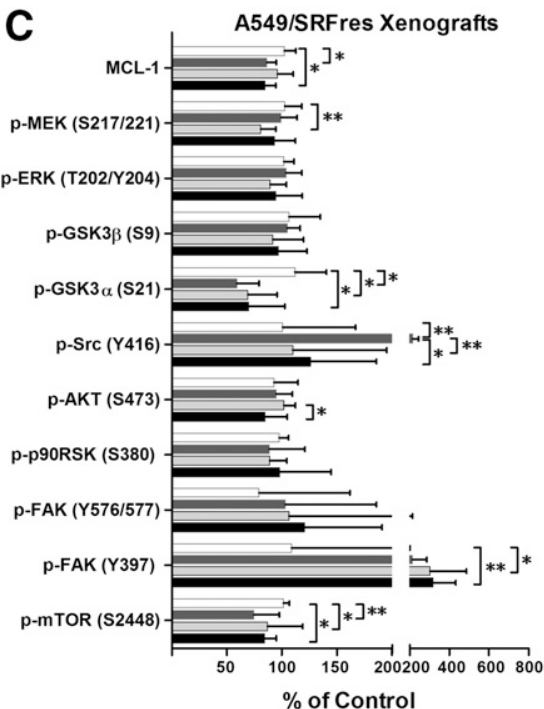

D

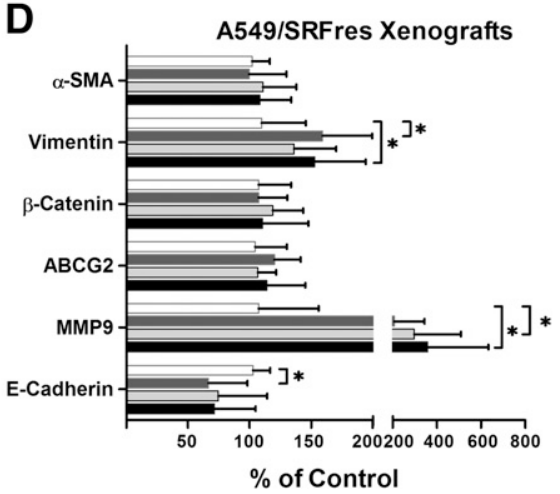

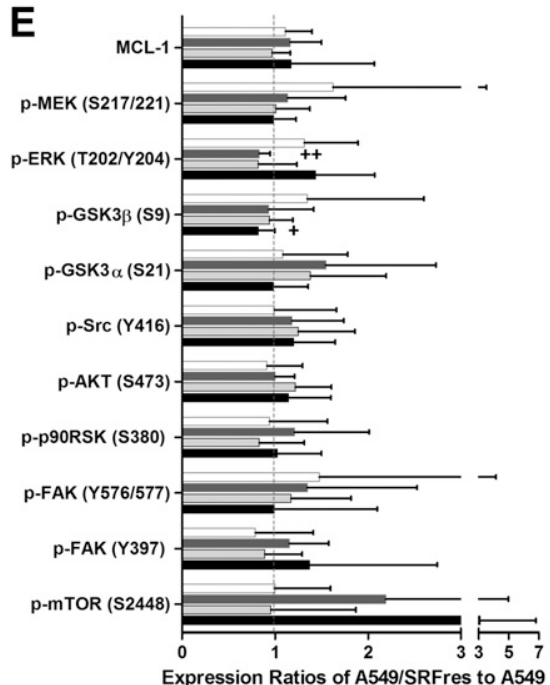

$\mathbf{F}$

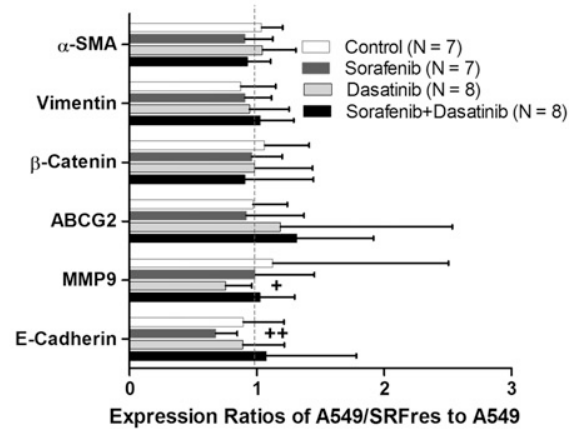

Fig 6 Western blot analyses were performed to compare protein expression levels among individual study groups and between matched pairs of A549 and A549/SRFres tumors. (A-D) Quantification of Western blots by image densitometry demonstrated that sorafenib-dasatinib combination therapy blocks AKT and Src phosphorylation, which is induced by sorafenib monotherapy in A549 and A549/SRFres tumors, but fails to decrease the expression of phospho-FAK (Y397), MMP9, and vimentin. Relative immunoreactive band intensities are expressed as the percent change over the average signal value in vehicle control tumors, with normalization to $\beta$-actin loading controls. For phosphorylated proteins, results are expressed as the ratio of phosphorylated to total species relative to the control tumor. (E and F) Comparison between the matched pairs of A549 and A549/SRFres tumors reveals significant decrease in expression of phospho-ERK and E-cadherin in sorafenib-treated A549/SRFres tumors and significant decrease in MMP9 and phospho-GSK3 $\beta$ in dasatinib- and combination-treated A549/SRFres tumors, respectively. Values indicate the mean ratio of protein expression level in A549/SRFres tumor to that in A549 tumor \pm S.D. Error bars are S.D. $* \mathrm{P}<0.05$; $* * \mathrm{P}<0.01$ using Kruskal-Wallis one-way analysis of variance on ranks, followed by the post hoc Kruskal-Wallis multiple comparison $\mathrm{z}$-value test. $+\mathrm{P}<0.05 ;++\mathrm{P}<0.01$ compared with the counterpart A549 xenografts using the paired sample t test.

1.7-fold lower than sorafenib tumor concentrations irrespective of tumor types (Table 1).

\section{Discussion}

Although considerable efforts have been made to investigate mechanisms underpinning the acquired sorafenib resistance in various types of cancer, none has focused on NSCLC. In this study, an A549 human lung adenocarcinoma xenograft model was used to explore the potential mechanism of acquired sorafenib resistance. In vivo drug-resistant models are more advantageous than in vitro models because they provide the tissue microenvironment in which tumor cells reside. The model used in this study was established using a sorafenibresistant A549 subline derived from the fastest growing A549 tumor that has become insensitive to sorafenib treatment in vivo. Compared with serial in vivo passaging of primary tumors, using the sorafenib-resistant A549 subline with low passage numbers $(<10)$ has the advantage of providing reproducible insights into the biologically relevant mechanism underlying the resistance and facilitating the process of identifying effective therapeutic strategy to overcome the resistance, and yet it suffers the drawback of possible changes in certain genetic features of primary tumors during the in vivo, to in vitro, to in vivo transition. An intriguing observation of our in vivo study was that A549/SRFres xenografts exhibited significantly rapid growth compared with their corresponding parental counterparts (Figs. 1D and 4D), even though the doubling times of cultured A549/ SRFres cells were similar to those of A549 cells. This finding raises the possibility that the prior selection process enables A549/SRFres cells to acquire certain traits that allow them to adapt to the microenvironmental proliferation barriers more quickly than A549 parental cells. Despite the differential tumor growth rate observed in vivo, we used this model to identify a new bypass mechanism of acquired sorafenib resistance and determine whether dasatinib can be a potential adjunct to sorafenib therapy to overcome the resistance. 
TABLE 1

Sorafenib and dasatinib concentrations in plasma and tumors 4 hours after the last dose Data are presented as mean \pm S.D.

\begin{tabular}{|c|c|c|c|}
\hline & $\begin{array}{c}\text { Dasatinib (40 mg/kg daily) } \\
\mathrm{n}=8\end{array}$ & $\begin{array}{c}\text { Sorafenib (40 mg/kg daily) } \\
\mathrm{n}=7\end{array}$ & $\begin{array}{c}\text { Dasatinib (20 mg/kgdaily) + } \\
\text { Sorafenib (20 mg/kg daily) } \\
n=8\end{array}$ \\
\hline \multicolumn{4}{|c|}{$C_{4 \mathrm{~h}, \text { plasma }}(\mu \mathrm{M})$} \\
\hline Dasatinib & $1.16 \pm 0.32$ & & $0.67 \pm 0.48$ \\
\hline Sorafenib & & $13.85 \pm 4.04$ & $5.38 \pm 2.53$ \\
\hline \multicolumn{4}{|c|}{$\mathrm{C}_{4 \mathrm{~h}, \mathrm{~A} 549 \text { xenograft }}(\mu \mathrm{M})$} \\
\hline Dasatinib & $2.70 \pm 1.35$ & & $1.74 \pm 0.59$ \\
\hline Sorafenib & & $7.74 \pm 2.83$ & $4.15 \pm 1.36$ \\
\hline \multicolumn{4}{|c|}{$\mathrm{C}_{4 \mathrm{~h}, \mathrm{~A} 549 \text { xenograft: }} \mathrm{C}_{4 \mathrm{~h}, \text { plasma }}$ ratio } \\
\hline Dasatinib & $2.32 \pm 4.27$ & & $3.18 \pm 1.54$ \\
\hline Sorafenib & & $0.57 \pm 0.20$ & $0.84 \pm 0.33$ \\
\hline \multicolumn{4}{|c|}{$\mathrm{C}_{4 \mathrm{~h}, \mathrm{~A} 549 / \mathrm{SRF} \text { res xenograft }}(\mu \mathrm{M})$} \\
\hline Dasatinib & $3.34 \pm 1.74$ & & $1.82 \pm 0.68$ \\
\hline Sorafenib & & $9.39 \pm 4.39$ & $3.24 \pm 1.89$ \\
\hline \multicolumn{4}{|c|}{$\mathrm{C}_{4 \mathrm{~h}, \mathrm{~A} 549 / \mathrm{SRFres} \text { xenograft }}: \mathrm{C}_{4 \mathrm{~h} \text {,plasma }}$ ratio } \\
\hline $\begin{array}{l}\text { Dasatinib } \\
\text { Dasating }\end{array}$ & $\begin{array}{l}2.87 \pm 5.51 \\
2.51\end{array}$ & & $3.24 \pm 1.59$ \\
\hline Sorafenib & & $0.69 \pm 0.33$ & $0.61 \pm 0.32$ \\
\hline
\end{tabular}

Examination of the expression pattern of several EMT markers in A549 and A549/SRFres xenografts revealed the tendency of downregulated E-cadherin and upregulated vimentin expression in sorafenib-treated tumors compared with controls (Figs. 2B; 6, B and C). Sorafenib-treated A549/ SRFres xenografts appeared to undergo EMT events similar to those of sorafenib-treated A549 xenografts but to a greater extent (Fig. 6F). EMT, activation of which often results in aggressive tumor behaviors, has been associated with resistance to sorafenib in hepatocellular carcinoma (HCC) models. In a recent study, the in vitro established sorafenibresistant HepG2 and Hus7S1 cell lines displayed a loss of E-cadherin and an increase in vimentin expression, accompanied by an increased invasive potential (van Malenstein et al., 2013). In this study, no marked difference in the expression of selected EMT markers was observed between cultured A549 and A549/SRFres cells, nor was any difference found in their migratory and invasive phenotype irrespective of the treatment (Fig. 3), suggesting the crucial role of tumor microenvironment in inducing the EMT (Jing et al., 2011). The exact mechanism accounting for the significant decrease in $\beta$-catenin levels in both sorafenib-treated A549 and A549/SRFres tumors is unclear. Nonetheless, it has been reported that loss or downregulation of $\beta$-catenin was associated with disease progress in malignant melanoma (Kageshita et al., 2001), and sorafenib was effective in decreasing $\beta$-catenin protein levels in human liver cancer cell lines (Lachenmayer et al., 2012). Therefore, we speculate that the decreased $\beta$-catenin levels in tumor tissue homogenates might be due to the decreased membranous and cytoplasmic $\beta$-catenin levels resulting from the loss of interaction between E-cadherin and $\beta$-catenin that potentially releases $\beta$-catenin from cell adherens junctions (Onder et al., 2008) followed by the nuclear translocation of $\beta$-catenin, leading to the induction of EMT (Alvarado et al., 2011; Ghahhari and Babashah, 2015).

In this study, besides the significant upregulation of MMP9 protein expression in sorafenib-treated A549/SRFres tumors, we also observed elevated expression levels of phospho-AKT (S473), phospho-FAK (Y397), and phospho-Src (Y416) in $40 \mathrm{mg} / \mathrm{kg}$ per day sorafenib-treated A549 and A549/SRFres tumors with no statistical significance, in part owing to the large interindividual variability. Nonetheless, our in vitro study demonstrated that the baseline phospho-Src (Y416) and phospho-FAK (Y576/577) levels in A549/SRFres cells were markedly greater than those in A549 cells (Fig. 3C). Given that activation of FAK and AKT signaling pathways has been suggested to upregulate the MMP9 expression in HCC, leading to enhanced cell invasion (Cheng et al., 2006; Chen et al., 2010a), it is tempting to speculate that AKT, FAK, and Src are potential alternate signaling molecules being activated to evade sorafenib therapy. Compensatory activation of the PI3K/AKT signaling pathway has been reported to mediate acquired sorafenib resistance (Chen et al., 2011), and therapeutic synergy could be achieved by combining sorafenib with PI3K/AKT inhibitors (Chen et al., 2010b; Zhai et al., 2015; Lindblad et al., 2016). In addition, activation of PI3K/AKT pathway downregulates membranous E-cadherin and $\beta$-catenin levels and promotes tumor cell invasion (Yip and Seow, 2012), suggesting that the observed downregulation of E-cadherin and $\beta$-catenin in sorafenib-treated tumors may attribute to sorafenib-induced activation of PI3K/AKT pathway. Although sorafenib-induced activation of FAK and Src has not been documented so far, the possible connection between FAK and acquired sorafenib resistance has been described in a recent study, which showed that the $\alpha \mathrm{v} \beta 3$ integrin/FAK/PI3K/AKT signaling pathway was involved in galectin-1-induced EMT and sorafenib resistance in HCC cells (Zhang et al., 2016). FAK and Src are cytoplasmic nonreceptor tyrosine kinases influencing cell adhesion by their direct or indirect effects on other adhesion regulators (Calautti et al., 1998; Noren et al., 2001). FAK is a downstream target of several growth factors (Mitra et al., 2005), whereas Src is a crucial mediator of FAK-regulated processes (Westhoff et al., 2004). The FAK-Src complex is activated through integrin-stimulated FAK phosphorylation at Y397, which creates a binding site for Src. Src, in turn, mediates phosphorylation of Y576/Y577 in the FAK domain activation loop, which subsequently activates the p130Cas-associated motility-promoting signaling cascades, leading to the induction and activation of MMP2 and MMP9 that promote cancer cell invasion (Van Slambrouck et al., 2007).

Based on our initial findings, we proposed an alternative survival mechanism that would allow the KRAS-mutant NSCLC to escape from sorafenib therapy through the activation 
of FAK/Src complex. Given the essential role of Src in mediating FAK phosphorylation and driving tumor cell proliferation, survival, adhesion, motility, and invasion (Bromann et al., 2004; Thomas and Jordan, 2004), we next investigated whether sorafenib in combination with dasatinib could increase antitumor efficacy and delay the onset of acquired sorafenib resistance. Results of the in vitro study showed that simultaneous exposure to sorafenib and dasatinib at fixed molar ratios of 3:1, $1: 1$, and 1:3 produced mostly additive to synergistic cytotoxic effects in both A549 and A549/SRFres cells, suggesting that simultaneous administration of sorafenib and dasatinib is an appropriate schedule for this combination. Determination of sorafenib and dasatinib concentrations in plasma and tumors demonstrated that the intratumoral concentration ratio of sorafenib to dasatinib was about 3 , which was within the range of concentration ratio that had produced synergistic effect in vitro. In agreement with the in vitro data (Fig. 4, A and B), it appeared that dasatinib alone or in combination with sorafenib resulted in a relatively greater tumor growth inhibition in A549/SRFres tumors than that in A549 tumors (Fig. 4, C and D). The augmented susceptibility of A549/SRFres tumors to dasatinib treatment may be associated with the upregulated baseline FAK-Src activity in A549/SRFres cells (Fig. 3C). Despite the evidence of in vitro synergy between sorafenib and dasatinib, the in vivo antitumor effect of half-dose sorafenib-dasatinib combination therapy was inferior to that of the full-dose sorafenib treatment but superior to that of the fulldose dasatinib treatment, regardless of tumor types. Nonetheless, the half-dose sorafenib-dasatinib combination exhibited a greater antitumor activity than single-agent sorafenib or dasatinib used at the same dose. As indicated by the Western blotting analysis, although the half-dose combination effectively inhibited Src and AKT phosphorylation, it did not block Y576/577-FAK phosphorylation nor decrease vimentin expression in tumors, but it unexpectedly increased the Y397-FAK phosphorylation and intratumoral MMP9 expression (Fig. 6), suggesting that inhibition of Src phosphorylation alone is insufficient to impede the activation of EMT and elevation of invasive potential associated with acquired sorafenib resistance. The upregulated Y397-FAK phosphorylation is possibly triggered by microenvironmental cues such as cytokines, growth factors, integrins, and so on (Sulzmaier et al., 2014). Further studies are needed to characterize the impact of tumor microenvironment on the Src-independent FAK signal transduction pathway.

Taken together, results of this study revealed not only the contribution of FAK/Src activation to the acquired sorafenib resistance in KRAS-mutant A549 xenografts by promoting the EMT and invasive potential but also the fact that Src inhibition alone was insufficient to overcome the resistance. Given the evolving redundancy of oncogenic pathways that enable tumor cells to compensate for several targeted genes and pathways, the major challenge of overcoming acquired resistance to pathway-targeted drugs is to identify a "master switch" that regulates and coordinates multiple signaling pathways involved in the resistance. Nonetheless, this work provides an example of rational combination of two targeted therapeutic agents to combat the acquired tumor resistance to single-agent targeted therapy. Based on our findings, further investigation is warranted to identify the most effective combination therapy to maximize the therapeutic benefit of sorafenib in KRAS-mutant NSCLC.

\section{Authorship Contributions}

Participated in research design: Zhou.

Conducted experiments: Guo, Choksi, Zhou.

Performed data analysis: Guo, Zhou.

Wrote or contributed to the writing of the manuscript: Zhou, Guo.

\section{References}

Alvarado CG, Maruyama S, Cheng J, Ida-Yonemochi H, Kobayashi T, Yamazaki M, Takagi R, and Saku T (2011) Nuclear translocation of $\beta$-catenin synchronized with loss of E-cadherin in oral epithelial dysplasia with a characteristic two-phase appearance. Histopathology 59:283-291.

Blumenschein GR, Jr, Saintigny P, Liu S, Kim ES, Tsao AS, Herbst RS, Alden C, Lee JJ, Tang X, Stewart DJ, et al. (2013) Comprehensive biomarker analysis and fina efficacy results of sorafenib in the BATTLE trial. Clin Cancer Res 19:6967-6975.

Bromann PA, Korkaya H, and Courtneidge SA (2004) The interplay between Src family kinases and receptor tyrosine kinases. Oncogene 23:7957-7968.

Calautti E, Cabodi S, Stein PL, Hatzfeld M, Kedersha N, and Paolo Dotto G (1998) Tyrosine phosphorylation and src family kinases control keratinocyte cell-cell adhesion. J Cell Biol 141:1449-1465.

Chan BA and Hughes BG (2015) Targeted therapy for non-small cell lung cancer: current standards and the promise of the future. Transl Lung Cancer Res 4:36-54 Chen JS, Huang XH, Wang Q, Chen XL, Fu XH, Tan HX, Zhang LJ, Li W, and Bi J (2010a) FAK is involved in invasion and metastasis of hepatocellular carcinoma. Clin Exp Metastasis 27:71-82.

Chen KF, Chen HL, Tai WT, Feng WC, Hsu CH, Chen PJ, and Cheng AL (2011) Activation of phosphatidylinositol 3-kinase/Akt signaling pathway mediates acquired resistance to sorafenib in hepatocellular carcinoma cells. J Pharmacol Exp Ther 337:155-161.

Chen KF, Yu HC, Liu TH, Lee SS, Chen PJ, and Cheng AL (2010b) Synergistic interactions between sorafenib and bortezomib in hepatocellular carcinoma involve PP2A-dependent Akt inactivation. J Hepatol 52:88-95.

Cheng JC, Chou CH, Kuo ML, and Hsieh CY (2006) Radiation-enhanced hepatocellular carcinoma cell invasion with MMP-9 expression through PI3K/Akt/NFkappaB signal transduction pathway. Oncogene 25:7009-7018.

Chou TC and Talalay P (1981) Generalized equations for the analysis of inhibitions of Michaelis-Menten and higher-order kinetic systems with two or more mutually exclusive and nonexclusive inhibitors. Eur J Biochem 115:207-216.

Dingemans AM, Mellema WW, Groen HJ, van Wijk A, Burgers SA, Kunst PW, Thunnissen E, Heideman DA, and Smit EF (2013) A phase II study of sorafenib in patients with platinum-pretreated, advanced (Stage IIIb or IV) non-small cell lung cancer with a KRAS mutation. Clin Cancer Res 19:743-751.

Firtina Karagonlar Z, Koc D, Iscan E, Erdal E, and Atabey N (2016) Elevated hepatocyte growth factor expression as an autocrine c-Met activation mechanism in acquired resistance to sorafenib in hepatocellular carcinoma cells. Cancer Sci 107 407-416.

Ghahhari NM and Babashah S (2015) Interplay between microRNAs and WNT/ $\beta$-catenin signalling pathway regulates epithelial-mesenchymal transition in cancer. Eur J Cancer 51:1638-1649.

Götz R (2008) Inter-cellular adhesion disruption and the RAS/RAF and beta-catenin signalling in lung cancer progression. Cancer Cell Int 8:7.

Harada K, Miyake H, Kusuda Y, and Fujisawa M (2014) Characterization of mechanism involved in acquired resistance to sorafenib in a mouse renal cell cancer RenCa model. Clin Transl Oncol 16:801-806.

Jing Y, Han Z, Zhang S, Liu Y, and Wei L (2011) Epithelial-mesenchymal transition in tumor microenvironment. Cell Biosci 1:29.

Kageshita T, Hamby CV, Ishihara T, Matsumoto K, Saida T, and Ono T (2001) Loss of beta-catenin expression associated with disease progression in malignant melanoma. Br J Dermatol 145:210-216.

Kantarjian H, Jabbour E, Grimley J, and Kirkpatrick P (2006) Dasatinib. Nat Rev Drug Discov 5:717-718.

Kim ES, Herbst RS, Wistuba II, Lee JJ, Blumenschein GR, Jr, Tsao A, Stewart DJ, Hicks ME, Erasmus J, Jr, Gupta S, et al. (2011) The BATTLE trial: personalizing therapy for lung cancer. Cancer Discov 1:44-53.

Lachenmayer A, Alsinet C, Savic R, Cabellos L, Toffanin S, Hoshida Y, Villanueva A, Minguez B, Newell P, Tsai HW, et al. (2012) Wnt-pathway activation in two molecular classes of hepatocellular carcinoma and experimental modulation by sorafenib. Clin Cancer Res 18:4997-5007.

Lindblad O, Cordero E, Puissant A, Macaulay L, Ramos A, Kabir NN, Sun J, VallonChristersson J, Haraldsson K, Hemann MT, et al. (2016) Aberrant activation of the $\mathrm{PI} 3 \mathrm{~K} / \mathrm{m}$ TOR pathway promotes resistance to sorafenib in AML. Oncogene 35 : 5119-5131

Liu L, Cao Y, Chen C, Zhang X, McNabola A, Wilkie D, Wilhelm S, Lynch M, and Carter C (2006) Sorafenib blocks the RAF/MEK/ERK pathway, inhibits tumor angiogenesis, and induces tumor cell apoptosis in hepatocellular carcinoma model PLC/PRF/5. Cancer Res 66:11851-11858.

Mahoney CL, Choudhury B, Davies H, Edkins S, Greenman C, Haaften Gv, Mironenko T, Santarius T, Stevens C, Stratton MR, et al. (2009) LKB1/KRAS mutant lung cancers constitute a genetic subset of NSCLC with increased sensitivity to MAPK and mTOR signalling inhibition. Br J Cancer 100:370-375.

McMillin DW, Negri JM, and Mitsiades CS (2013) The role of tumour-stromal interactions in modifying drug response: challenges and opportunities. Nat Rev Drug Discov 12:217-228.

Mitra SK, Hanson DA, and Schlaepfer DD (2005) Focal adhesion kinase: in command and control of cell motility. Nat Rev Mol Cell Biol 6:56-68.

Morgillo F, Cascone T, D'Aiuto E, Martinelli E, Troiani T, Saintigny P, De Palma R, Heymach JV, Berrino L, Tuccillo C, et al. (2011) Antitumour efficacy of MEK inhibitors in human lung cancer cells and their derivatives with acquired resistance to different tyrosine kinase inhibitors. Br J Cancer 105:382-392. 
Noren NK, Niessen CM, Gumbiner BM, and Burridge K (2001) Cadherin engagement regulates Rho family GTPases. J Biol Chem 276:33305-33308.

Onder TT, Gupta PB, Mani SA, Yang J, Lander ES, and Weinberg RA (2008) Loss of E-cadherin promotes metastasis via multiple downstream transcriptional pathways. Cancer Res 68:3645-3654.

Pao W and Chmielecki J (2010) Rational, biologically based treatment of EGFRmutant non-small-cell lung cancer. Nat Rev Cancer 10:760-774.

Paz-Ares L, Hirsh V, Zhang L, de Marinis F, Yang JC, Wakelee HA, Seto T, Wu YL, Novello S, Juhasz E, et al. (2015) Monotherapy administration of sorafenib in patients with non-small cell lung cancer (MISSION) trial: a phase III, multicenter, placebo-controlled trial of sorafenib in patients with relapsed or refractory predominantly nonsquamous non-small-cell lung cancer after 2 or 3 previous treatment regimens. J Thorac Oncol 10:1745-1753.

Rodriguez FJ, Lewis-Tuffin LJ, and Anastasiadis PZ (2012) E-cadherin's dark side: possible role in tumor progression. Biochim Biophys Acta 1826:23-31.

Sulzmaier FJ, Jean C, and Schlaepfer DD (2014) FAK in cancer: mechanistic findings and clinical applications. Nat Rev Cancer 14:598-610.

Sun X, Niu X, Chen R, He W, Chen D, Kang R, and Tang D (2016) Metallothionein-1G facilitates sorafenib resistance through inhibition of ferroptosis. Hepatology 64 488-500.

Tai WT, Cheng AL, Shiau CW, Liu CY, Ko CH, Lin MW, Chen PJ, and Chen KF (2012) Dovitinib induces apoptosis and overcomes sorafenib resistance in hepatocellular carcinoma through SHP-1-mediated inhibition of STAT3. Mol Cancer Ther 11:452-463.

Thamilselvan V, Craig DH, and Basson MD (2007) FAK association with multiple signal proteins mediates pressure-induced colon cancer cell adhesion via a Srcdependent PI3K/Akt pathway. FASEB J 21:1730-1741.

Thomas SM and Jordan TR (2004) Contributions of oral and extraoral facial movement to visual and audiovisual speech perception. J Exp Psychol Hum Percept Perform 30:873-888.

Tovar V, Cornella H, Moeini A, Vidal S, Hoshida Y, Sia D, Peix J, Cabellos L, Alsinet C, Torrecilla S, et al. (2017) Tumour initiating cells and IGF/FGF signalling contribute to sorafenib resistance in hepatocellular carcinoma. Gut 66:530-540.

Ulivi P, Arienti C, Amadori D, Fabbri F, Carloni S, Tesei A, Vannini I, Silvestrini R, and Zoli W (2009) Role of RAF/MEK/ERK pathway, p-STAT-3 and Mcl-1 in sorafenib activity in human pancreatic cancer cell lines. J Cell Physiol 220:214-221. van Malenstein H, Dekervel J, Verslype C, Van Cutsem E, Windmolders P, Nevens F, and van Pelt J (2013) Long-term exposure to sorafenib of liver cancer cells induces resistance with epithelial-to-mesenchymal transition, increased invasion and risk of rebound growth. Cancer Lett 329:74-83.
Van Slambrouck S, Grijelmo C, De Wever O, Bruyneel E, Emami S, Gespach C, and Steelant WF (2007) Activation of the FAK-src molecular scaffolds and p130Cas-JNK signaling cascades by alpha1-integrins during colon cancer cell invasion. Int J Oncol 31:1501-1508.

Wang H, Xu L, Zhu X, Wang P, Chi H, and Meng Z (2014) Activation of phosphatidylinositol 3-kinase/Akt signaling mediates sorafenib-induced invasion and metastasis in hepatocellular carcinoma. Oncol Rep 32:1465-1472.

Westhoff MA, Serrels B, Fincham VJ, Frame MC, and Carragher NO (2004) SRCmediated phosphorylation of focal adhesion kinase couples actin and adhesion dynamics to survival signaling. Mol Cell Biol 24:8113-8133.

Wheeler DL, Dunn EF, and Harari PM (2010) Understanding resistance to EGFR inhibitors-impact on future treatment strategies. Nat Rev Clin Oncol 7:493-507.

Wilhelm S, Carter C, Lynch M, Lowinger T, Dumas J, Smith RA, Schwartz B, Simantov R, and Kelley S (2006) Discovery and development of sorafenib: a multikinase inhibitor for treating cancer. Nat Rev Drug Discov 5:835-844.

Wilhelm SM, Carter C, Tang L, Wilkie D, McNabola A, Rong H, Chen C, Zhang X, Vincent P, McHugh M, et al. (2004) BAY 43-9006 exhibits broad spectrum oral antitumor activity and targets the RAF/MEK/ERK pathway and receptor tyrosine kinases involved in tumor progression and angiogenesis. Cancer Res 64:7099-7109.

Yip WK and Seow HF (2012) Activation of phosphatidylinositol 3-kinase/Akt signaling by EGF downregulates membranous E-cadherin and $\beta$-catenin and enhances invasion in nasopharyngeal carcinoma cells. Cancer Lett 318:162-172.

Zhai B, Hu F, Yan H, Zhao D, Jin X, Fang T, Pan S, Sun X, and Xu L (2015) Bufalin reverses resistance to sorafenib by inhibiting Akt activation in hepatocellular carcinoma: the role of endoplasmic reticulum stress. PLoS One 10:e0138485.

Zhang PF, Li KS, Shen YH, Gao PT, Dong ZR, Cai JB, Zhang C, Huang XY, Tian MX, $\mathrm{Hu} \mathrm{ZQ}$, et al. (2016) Galectin-1 induces hepatocellular carcinoma EMT and sorafenib resistance by activating FAK/PI3K/AKT signaling. Cell Death Dis 7:e2201.

Zhou Q, Guo P, and Gallo JM (2008) Impact of angiogenesis inhibition by sunitinib on tumor distribution of temozolomide. Clin Cancer Res 14:1540-1549.

Zhou Q, Lv H, Mazloom AR, Xu H, Ma'ayan A, and Gallo JM (2012) Activation of alternate prosurvival pathways accounts for acquired sunitinib resistance in U87MG glioma xenografts. J Pharmacol Exp Ther 343:509-519.

Address correspondence to: Qingyu Zhou, University of South Florida College of Pharmacy, 12901 Bruce B. Downs Boulevard, MDC30, Tampa, FL 33612. E-mail: qzhou1@health.usf.edu 